\title{
Control theorems for $\ell$-adic Lie extensions of global function fields
}

\author{
ANDREA BANDINI AND MARIA VALENTINO
}

\begin{abstract}
Let $F$ be a global function field of characteristic $p>0, K / F$ an $\ell$-adic Lie extension unramified outside a finite set of places $S$ and $A / F$ an abelian variety. We study $\operatorname{Sel}_{A}(K)_{\ell}^{\vee}$ (the Pontrjagin dual of the Selmer group) and (under some mild hypotheses) prove that it is a finitely generated $\mathbb{Z}_{\ell}[[\mathrm{Gal}(K / F)]]$ module via generalizations of Mazur's Control Theorem. If $\mathrm{Gal}(K / F)$ has no elements of order $\ell$ and contains a closed normal subgroup $H$ such that $\operatorname{Gal}(K / F) / H \simeq \mathbb{Z}_{\ell}$, we are able to give sufficient conditions for $\operatorname{Sel}_{A}(K)_{\ell}^{\vee}$ to be finitely generated as $\mathbb{Z}_{\ell}[[H]]$-module and, consequently, a torsion $\mathbb{Z}_{\ell}[[\operatorname{Gal}(K / F)]]$-module. We deal with both cases $\ell \neq p$ and $\ell=p$.
\end{abstract}

Mathematics Subject Classification (2010): 11R23 (primary); 11R58, 11G35 (secondary).

\section{Introduction}

One of the main features of Iwasawa theory is the link it provides between characteristic ideals of (duals of) Selmer groups and $p$-adic $L$-functions. In the classical abelian setting of $\mathbb{Z}_{\ell}^{d}$-extensions both its analytic and algebraic sides have been well developed for general global fields, leading to the statements (and, in some cases, to the proofs) of Main Conjectures. The foundations for the research in noncommutative Iwasawa theory for a general $\ell$-adic Lie extension can be found in the celebrated paper by Coates et al. [9] and the subject has been developed from there, first in the number field setting and (more recently) for function fields of positive characteristic.

In this work we deal with the function field setting. Namely let $F$ be a global function field of trascendence degree one over its constant field $\mathbb{F}_{q}$, where $q$ is a power of a fixed prime $p \in \mathbb{Z}$. Let $K$ be a Galois extension of $F$ unramified outside a finite set of primes $S$ and such that $G=\operatorname{Gal}(K / F)$ is an infinite $\ell$-adic Lie group ( $\ell \in \mathbb{Z}$ a prime number). Let $\Lambda(G)$ be the associated Iwasawa algebra (for precise definitions of all notations and objects appearing in this Introduction see Section 2). Finally, let $A$ be an abelian variety defined over $F$ and of finite dimension $g$. 
When $G$ has no elements of order $\ell$ and contains a closed normal subgroup $H$ such that $G / H \simeq \mathbb{Z}_{\ell}$, Coates et al. ([9]) are able to define a characteristic element for every $\Lambda(G)$-module $M$ which belongs to the category $\mathfrak{M}_{H}(G)$ : a finitely generated $\Lambda(G)$-module $M$ lies in the category $\mathfrak{M}_{H}(G)$ when the quotient of $M$ by the submodule of elements of order a finite power of $\ell$ is finitely generated over the Iwasawa algebra of $H$. Moreover, for the number field setting, they stated the following conjecture (which is [9, Conjecture 5.1]):

Conjecture 1.1. Let $F$ be a number field and $E / F$ an elliptic curve with good ordinary reduction at $p \geqslant 5$. Put $F_{\infty}:=F\left(E\left[p^{\infty}\right]\right), \mathbb{Q}^{\text {cyc }}$ as the cyclotomic $\mathbb{Z}_{p^{-}}$ extension of $\mathbb{Q}$ and $H=\operatorname{Gal}\left(F_{\infty} / \mathbb{Q}^{\text {cyc }}\right)$. Then, $\operatorname{Sel}_{E}\left(F_{\infty}\right)_{p}^{\vee}$ belongs to the category $\mathfrak{M}_{H}(G)$.

In order to generalize this statement to our extensions $K / F$, we shall study the structure of $\operatorname{Sel}_{A}(K)_{\ell}^{\vee}$ (the Pontrjagin dual of the Selmer group) as a module over both $\Lambda(G)$ and $\Lambda(H)$ via the classical tool provided by Mazur's Control Theorem (see [19] and, for recent generalizations to $\mathbb{Z}_{\ell}^{d}$-extensions of function fields, [3-5] and [34]). We will prove that $\operatorname{Sel}_{A}(K)_{\ell}^{\vee}$ is a finitely generated $\Lambda(G)$-module and, in a similar way, we obtain that $\operatorname{Sel}_{A}(K)_{\ell}^{\vee}$ is a finitely generated $\Lambda(H)$-module as well, provided that $G$ contains a suitable closed subgroup $H$ (and, for $\ell=p$, under

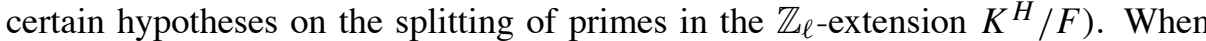
this is the case, since $H$ has infinite index in $G, \operatorname{Sel}_{A}(K)_{\ell}^{\vee}$ is also a torsion $\Lambda(G)$ module. For some related results regarding the presence of pseudo-null submodules in $\operatorname{Sel}_{A}(K)_{\ell}^{\vee}$ (for $\ell \neq p$ ), see [7].

Working on the algebra $\Lambda(H)$ is often sufficient to gather information about $\operatorname{Sel}_{A}(K)_{\ell}^{\vee}$ as a $\Lambda(G)$-module, but we prefer to work on $\Lambda(G)$ as well for several reasons. First of all, as one can see in [9], we always need finitely generated $\Lambda(G)$ modules. Secondly, in our first control theorem (Theorem 3.2) it is possible to find the path we will follow and the tools we will use in most of the proofs of the main theorems (including the ones for $\Lambda(H)$-modules involving the extensions $K / K^{H}$, which are not, strictly speaking, control theorems since the base field will not be allowed to vary). Finally, control theorems for $\Lambda(G)$-modules sometimes allow us to obtain results on general $\ell$-adic Lie extensions, regardless of $G$ containing or not an arithmetically interesting subgroup $H$.

We recall that recent papers have considered similar and/or more general topics using different (somehow more sophisticated) techniques. For example, in [23], the authors study the structure of Selmer groups using syntomic cohomology for $p$ adic Lie extensions of fields of characteristic $p$ containing the unique $\mathbb{Z}_{p}$-extension of the constant field. Moreover [38] provides a comprehensive study of the whole theory (including a proof of the main conjecture) in the language of schemes, for $\ell$-adic extensions of a separated scheme $X$ of finite type over the field $\mathbb{F}_{p^{e}}$; the proof uses K-theory, Waldhausen categories (and higher $K$-groups) and other cohomological tools. Another approach to the main conjecture (for $\mathbb{Z}_{p}^{d}$-extensions) is provided in [16].

Our method requires a careful study of Galois cohomology groups (both for local and global fields), hence control theorems, in addition to being interesting in 
their own right, provide what can be now considered as an "elementary approach" to the study of Selmer groups. Nevertheless this approach, fit also to investigate some issues of the analytic side of Iwasawa theory (as done in [25]), guarantees a lot of information on the structure of Selmer groups and, in particular, many cases in which an analogue of Conjecture 1.1 is verified (see Corollaries 4.9 and 6.5).

Here is a summary of the paper. After recalling (in Section 2) the main objects and the setting we will work with, we shall start with the case $\ell \neq p$ proving the control theorem in Section 3 and examining its consequences on the $\Lambda(G)$ structure of $\operatorname{Sel}_{A}(K)_{\ell}^{\vee}$ in Section 4. In particular we shall prove the following (see Theorem 3.2)

Theorem 1.2. For any finite extension $F^{\prime} / F$ contained in $K$, the kernels and cokernels of the maps

$$
a_{K / F^{\prime}}: \operatorname{Sel}_{A}\left(F^{\prime}\right)_{\ell} \longrightarrow \operatorname{Sel}_{A}(K)_{\ell}^{\mathrm{Gal}\left(K / F^{\prime}\right)}
$$

are cofinitely generated $\mathbb{Z}_{\ell}$-modules. If all primes in $S$ and all primes of bad reduction have decomposition groups open in $G$, then the coranks of kernels and cokernels are bounded independently of $F^{\prime}$. Moreover if $A\left[\ell^{\infty}\right](K)$ is finite, then such kernels and cokernels are of finite order.

An immediate application of Nakayama's Lemma will show that $\operatorname{Sel}_{A}(K)_{\ell}$ is a cofinitely generated $\Lambda(G)$-module. Then, in Section 4, we prove an analogous statement (Theorem 4.8) for the kernel and cokernel of the map

$$
a_{K / K^{\prime}}: \operatorname{Sel}_{A}\left(K^{\prime}\right)_{\ell} \longrightarrow \operatorname{Sel}_{A}(K)_{\ell}^{\mathrm{Gal}\left(K / K^{\prime}\right)},
$$

where $\operatorname{Gal}\left(K / K^{\prime}\right)=H$ and $G / H \simeq \mathbb{Z}_{\ell}$. We derive from that the structure of $\operatorname{Sel}_{A}(K)_{\ell}^{\vee}$ as a $\Lambda(H)$-module and, as a consequence, some cases in which $\operatorname{Sel}_{A}(K)_{\ell}^{\vee}$ is a torsion $\Lambda(G)$-module. We also deal with the case $K=F\left(A\left[\ell^{\infty}\right]\right)$ which can be included in this general case (with the only exception of the ad hoc result of Theorem 3.7).

In Sections 5 and 6 we follow the same path and obtain analogous results for the case $\ell=p$, where definitions and statements need the use of flat cohomology groups but most of the proofs only require the study of Galois cohomology groups (as in the $\ell \neq p$ case). The main difference will be the presence of nontrivial images for the Kummer maps in the definition of the Selmer groups. In particular we shall show (see Theorem 5.3)

Theorem 1.3. Assume that all ramified primes are of good ordinary or split multiplicative reduction, then, for any finite extension $F^{\prime} / F$ contained in $K$, the kernels and cokernels of the maps

$$
a_{K / F^{\prime}}: \operatorname{Sel}_{A}\left(F^{\prime}\right)_{p} \longrightarrow \operatorname{Sel}_{A}(K)_{p}^{\mathrm{Gal}\left(K / F^{\prime}\right)}
$$

are cofinitely generated $\mathbb{Z}_{p}$-modules. If all primes in $S$ and all primes of bad reduction have decomposition groups open in $G$, then the coranks of kernels and cokernels are bounded independently of $F^{\prime}$. 
As in the case $\ell \neq p$, we immediately find that $\operatorname{Sel}_{A}(K)_{p}^{\vee}$ is a finitely generated $\Lambda(G)$-module (this can be seen as the non-commutative counterpart of [34, Theorem 5]). Moreover we will prove that $\operatorname{Sel}_{A}(K)_{p}^{\vee}$ is often $\Lambda(G)$-torsion: when the ramified primes are of good ordinary reduction we provide a direct proof (see Theorem 6.2, which depends on the control Theorem 5.3, for a precise statement), when the ramified primes are of split multiplicative reduction we need to examine the structure of $\operatorname{Sel}_{A}(K)_{p}^{\vee}$ as a $\Lambda(H)$-module to get the result (see Corollary 6.5, which depends on the control Theorem 6.4).

We note that the extension $F\left(A\left[p^{\infty}\right]\right)$ is not included here because it should require the study of inseparable extensions which cannot be treated with the techniques used in this paper.

ACKnOWLEDGements. The authors thank David Burns, Ki-Seng Tan and Otmar Venjakob for useful discussions and comments on earlier drafts of this paper. We are grateful to John Coates for pointing out (and providing) the thesis [27]. We gratefully acknowledge the anonymous referee for comments which led to improvements in the exposition. The second named author wishes to thank the Mathematisches Institut of the Ruprecht-Karls Universität Heidelberg for hospitality and for providing a nice and challenging environment to start working on this project.

\section{Setting and notation}

For the convenience of the reader we recall here the main objects we shall deal with and give notation and definitions for them. The notations will be fairly standard for Iwasawa theory so the expert reader can simply skip this section and go back to it only when/if needed.

\subsection{Fields}

For any field $L$ we let $G_{L}=\operatorname{Gal}\left(L^{s} / L\right)\left(L^{s}\right.$ a separable closure of $\left.L\right)$ and $\mathcal{X}_{L}$ the scheme $\operatorname{Spec}(L)$ : they will essentially appear in Galois and flat cohomology groups so whenever we write a scheme $\mathcal{X}$ we always mean $\mathcal{X}_{f l}$ scheme on flat topology. Let $F$ be a global function field of trascendence degree one over its constant field $\mathbb{F}_{F}=\mathbb{F}_{q}$, where $q$ is a power of a fixed prime $p \in \mathbb{Z}$. We put $\bar{F}$ for an algebraic closure of $F$ and $F^{s} \subset \bar{F}$ for a separable closure.

For any algebraic extension $L / F$, let $\Sigma_{L}$ be the set of places of $L$ : for any $v \in \Sigma_{L}$ we let $L_{v}$ be the completion of $L$ at $v, \mathcal{O}_{v}$ its ring of integers with maximal ideal $\mathfrak{m}_{v}$ and residue field $\mathbb{F}_{L_{v}}$. Whenever we deal with a local field $E$ (or an algebraic extensions of such field) the above notations will often be replaced by $\mathcal{O}_{E}, \mathfrak{m}_{E}$ and $\mathbb{F}_{E}$.

For any place $v \in \Sigma_{F}$ we choose (and fix) an embedding $\bar{F} \hookrightarrow \overline{F_{v}}$ (an algebraic closure of $\left.F_{v}\right)$, in order to get a restriction map $G_{F_{v}}:=\operatorname{Gal}\left(\overline{F_{v}} / F_{v}\right) \hookrightarrow G_{F}$. 
All algebraic extensions of $F$ (respectively of $F_{v}$ ) will be assumed to be contained in $\bar{F}$ (respectively $\overline{F_{v}}$ ).

In general we will deal with $\ell$-adic Lie extensions $K / F$, i.e., Galois extensions with Galois group an $\ell$-adic Lie group. We always assume that our extensions are unramified outside a finite set $S$ of primes of $\Sigma_{F}$.

\section{2. $\ell$-adic Lie groups}

The most useful (for us) characterization of $\ell$-adic analytic groups is due to Lazard [17] and states that: a topological group $G$ has the structure of $\ell$-adic Lie group if and only if $G$ contains an open subgroup which is a uniform pro- $\ell$ group (see also [11, Theorem 8.32]).

For the whole paper our $\ell$-adic Lie group $G$ is the Galois group of a field extension, so one must take into account that it is also compact and profinite. Compactness implies it has finite rank and has an open, normal, uniform pro- $\ell$ subgroup which is always finitely generated (see [11, Corollary 8.34]). While being profinite means that every open subgroup is of finite index.

Finally, note that if $G$ is an $\ell$-adic Lie group without points of order $\ell$, then it has finite $\ell$-cohomological dimension, which is equal to its dimension as an $\ell$-adic Lie group ([28, Corollaire (1), page 413]).

For all basic definitions and facts about profinite groups, the reader is referred to [11] and [37].

\subsection{Modules and duals}

For any $\ell$-adic Lie group $G$ we denote by

$$
\Lambda(G)=\mathbb{Z}_{\ell}[[G]]:=\lim _{\overleftarrow{U}} \mathbb{Z}_{\ell}[G / U]
$$

the associated Iwasawa algebra (the limit is on the open normal subgroups of $G$ ). From Lazard's work (see [17]), we know that $\Lambda(G)$ is Noetherian and, if $G$ is pro$\ell$ and has no elements of order $\ell$, then $\Lambda(G)$ is an integral domain. From [11, Theorem 4.5] we know that, for a finitely generated powerful pro- $\ell$ group, being torsion free is equivalent to being uniform. Because of this when we need $\Lambda(G)$ to be without zero divisors we will take a torsion free $G$.

For a $\Lambda(G)$-module $M$, we denote its Pontrjagin dual by $M^{\vee}:=\operatorname{Hom}_{\text {cont }}\left(M, \mathbb{C}^{*}\right)$. In the cases considered in this paper, $M$ will be a (mostly discrete) topological

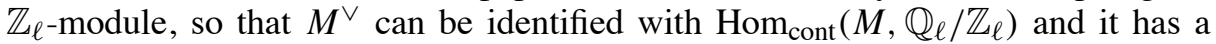
natural structure of $\mathbb{Z}_{\ell}$-module (because the category of compact $\Lambda(G)$-modules and the category of discrete $\Lambda(G)$-modules are both abelian and the Pontrjagin duality defines a contravariant equivalence of categories between them).

The reader is reminded that to say that an $R$-module $M$ ( $R$ any ring) is cofinitely generated over $R$ means that $M^{\vee}$ is a finitely generated $R$-module. 


\subsection{Selmer groups}

Let $A$ be an abelian variety of dimension $g$ defined over $F$ : we denote by $B$ its dual abelian variety. For any positive integer $n$ we let $A[n]$ be the scheme of $n$-torsion points and, for any prime $\ell$, we put $A\left[\ell^{\infty}\right]:=\lim _{\rightarrow} A\left[\ell^{n}\right]$.

We define Selmer groups via the usual cohomological techniques and, since we deal mostly with the flat scheme of torsion points, we shall use the flat cohomology groups $H_{f l}^{i}$ (for the basic definitions see [20, Chapter II and III]). Fix a prime $\ell \in \mathbb{Z}$ and consider the exact sequence

$$
0 \rightarrow A\left[\ell^{n}\right] \rightarrow A \stackrel{\ell^{n}}{\rightarrow} A \rightarrow 0
$$

and, for any finite algebraic extension $L / F$, take flat cohomology with respect to $\mathcal{X}_{L}$ to get an injective Kummer map

$$
A(L) / \ell^{n} A(L) \hookrightarrow H_{f l}^{1}\left(\mathcal{X}_{L}, A\left[\ell^{n}\right]\right)
$$

Taking direct limits one has an injective map

$$
\kappa: A(L) \otimes \mathbb{Q}_{\ell} / \mathbb{Z}_{\ell} \hookrightarrow \lim _{\vec{n}} H_{f l}^{1}\left(\mathcal{X}_{L}, A\left[\ell^{n}\right]\right):=H_{f l}^{1}\left(\mathcal{X}_{L}, A\left[\ell^{\infty}\right]\right)
$$

Exactly in the same way one can define local Kummer maps

$$
\kappa_{w}: A\left(L_{w}\right) \otimes \mathbb{Q}_{\ell} / \mathbb{Z}_{\ell} \hookrightarrow \lim _{n} H_{f l}^{1}\left(\mathcal{X}_{L_{w}}, A\left[\ell^{n}\right]\right):=H_{f l}^{1}\left(\mathcal{X}_{L_{w}}, A\left[\ell^{\infty}\right]\right)
$$

for any place $w \in \Sigma_{L}$.

Definition 2.1. The $\ell$-part of the Selmer group of $A$ over $L$ is defined to be

$$
\operatorname{Sel}_{A}(L)_{\ell}=\operatorname{Ker}\left\{H_{f l}^{1}\left(\mathcal{X}_{L}, A\left[\ell^{\infty}\right]\right) \rightarrow \prod_{w \in \Sigma_{L}} H_{f l}^{1}\left(\mathcal{X}_{L_{w}}, A\left[\ell^{\infty}\right]\right) / \operatorname{Im} \kappa_{w}\right\}
$$

where the map is the product of the natural restrictions between cohomology groups.

For infinite extensions $\mathcal{L} / F$ the $\operatorname{Selmer}_{\text {group }} \operatorname{Sel}_{A}(\mathcal{L})_{\ell}$ is defined, as usual, via direct limits.

Letting $L$ vary through subextensions of $K / F$, the groups $\operatorname{Sel}_{A}(L)_{\ell}$ admit natural actions by $\mathbb{Z}_{\ell}$ (because of $A\left[\ell^{\infty}\right]$ ) and by $G=\operatorname{Gal}(K / F)$. Hence they are modules over the Iwasawa algebra $\Lambda(G)$.

If $L / F$ is a finite extension the group $\operatorname{Sel}_{A}(L)_{\ell}$ is a cofinitely generated $\mathbb{Z}_{\ell^{-}}$ module (see, e.g. [21, III.8 and III.9]). One can define the Tate-Shafarevich group $\mathrm{III}(A / L)$ as the group that fits into the exact sequence

$$
A(L) \otimes \mathbb{Q}_{\ell} / \mathbb{Z}_{\ell} \hookrightarrow \operatorname{Sel}_{A}(L)_{\ell} \rightarrow \operatorname{III}(A / L)\left[\ell^{\infty}\right] .
$$


The function field version of the Birch and Swinnerton-Dyer conjecture predicts $\operatorname{III}(A / L)$ to be finite for any finite extension $L$ of $F$ (relevant results in this direction can be found, for example, in [15] and [26]). Assuming the BSD conjecture and taking Pontrjagin duals on the sequence above, one gets

$$
\operatorname{rank}_{\mathbb{Z}_{\ell}} \operatorname{Sel}_{A}(L)_{\ell}^{\vee}=\operatorname{rank}_{\mathbb{Z}} A(L)
$$

which provides motivation for the study of (duals of) Selmer groups (recall that the cohomology groups $H_{f l}^{i}$, hence the Selmer groups, are endowed with the discrete topology).

Remark 2.2. When $\ell \neq p$ the torsion subschemes are Galois modules and we can define Selmer groups via Galois cohomology since, in this case,

$$
H_{f l}^{1}\left(\mathcal{X}_{L}, A\left[\ell^{n}\right]\right) \simeq H_{e t}^{1}\left(\mathcal{X}_{L}, A\left[\ell^{n}\right]\right) \simeq H^{1}\left(G_{L}, A\left[\ell^{n}\right](\bar{F})\right)
$$

(see [20, III.3.9]). In order to lighten notations, each time we work with $\ell \neq p$ we shall use the classical notation $H^{i}(L, \cdot)$ instead of $H^{i}\left(G_{L}, \cdot\right)$ and $H^{i}(L / E, \cdot)$ instead of $H^{i}(\operatorname{Gal}(L / E), \cdot)$. Moreover we write $A[n]$ for $A[n](\bar{F})$, putting $A\left[\ell^{\infty}\right]:=$ $\bigcup A\left[\ell^{n}\right]$.

\subsection{The fundamental diagram}

We will consider $\ell$-adic Lie extensions $K / F^{\prime}$ (for any finite extension $F^{\prime} / F$ ) and study the kernels and cokernels of the natural restriction maps

$$
\operatorname{Sel}_{A}\left(F^{\prime}\right)_{\ell} \longrightarrow \operatorname{Sel}_{A}(K)_{\ell}^{\mathrm{Gal}\left(K / F^{\prime}\right)} .
$$

We shall do this via the snake lemma applied to the following diagram

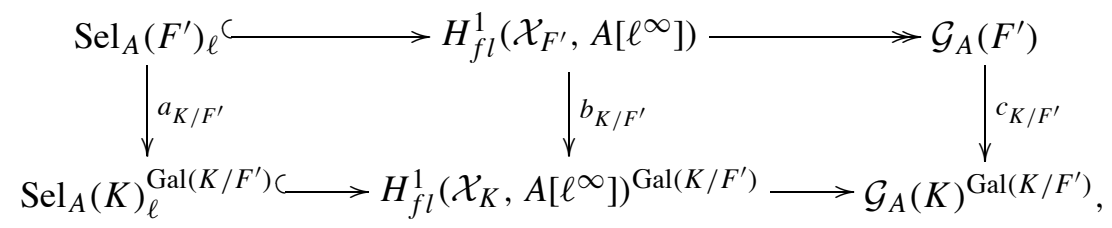

where, for any field $L$, we put

$$
\mathcal{G}_{A}(L)=\operatorname{Im}\left\{H_{f l}^{1}\left(\mathcal{X}_{L}, A\left[\ell^{\infty}\right]\right) \rightarrow \prod_{w \in \Sigma_{L}} H_{f l}^{1}\left(\mathcal{X}_{L_{w}}, A\left[\ell^{\infty}\right]\right) / \operatorname{Im} \kappa_{w}\right\}
$$

\section{Control theorem for $\ell \neq p$}

As pointed out by Coates and Greenberg in [10, Proposition 4.1 and the subsequent Remark], the image of the Kummer map $\kappa_{w}$ is trivial for any $w \in \Sigma_{L}$, because $L_{w}$ 
has characteristic $p \neq \ell$. Therefore, in this case, the $\ell$-part of the Selmer group is simply

$$
\operatorname{Sel}_{A}(L)_{\ell}=\operatorname{Ker}\left\{H^{1}\left(L, A\left[\ell^{\infty}\right]\right) \rightarrow \prod_{w \in \Sigma_{L}} H^{1}\left(L_{w}, A\left[\ell^{\infty}\right]\right)\right\} .
$$

The following lemma holds for any prime $\ell$ (including $\ell=p$ ) and we shall need it in most of our control theorems.

Lemma 3.1. Let $G$ be any compact $\ell$-adic Lie group of finite dimension and let $M$ be a discrete $\Lambda(G)$-module which is cofinitely generated over $\mathbb{Z}_{\ell}$ (respectively finite). Then, for any closed subgroup $\mathcal{V}$ of $G$, the cohomology groups $H^{1}(\mathcal{V}, M)$ and $H^{2}(\mathcal{V}, M)$ are cofinitely generated (respectively finite) $\mathbb{Z}_{\ell}$-modules as well and their coranks (respectively their orders) are bounded independently of $\mathcal{V}$.

Proof. Obviously we can restrict our attention to pro- $\ell$ subgroups of $G$ and, by [11, Chapter 1, Exercise 12], any group of this kind is contained in a pro- $\ell$ Sylow subgroup $P_{\ell}$. Moreover any pro- $\ell$ Sylow is open by [11, Corollary 8.34]. Let $\mathcal{V}$ be any closed pro- $\ell$ subgroup of some pro- $\ell$ Sylow $P_{\ell}$ and put

$$
d_{i}(\mathcal{V}):=\operatorname{dim}_{\mathbb{Z} / \ell \mathbb{Z}} H^{i}(\mathcal{V}, \mathbb{Z} / \ell \mathbb{Z}) \quad i=1,2 .
$$

Since $P_{\ell}$ has finite rank (in the sense of [11, Definition 3.12]) the cardinalities of a minimal set of topological generators for $\mathcal{V}$, i.e., the $d_{1}(\mathcal{V})$ 's, are all finite and bounded by $d_{1}\left(P_{\ell}\right)$. Moreover, since $P_{\ell}$ contains a uniform (see [11, Definition 4.1]) open subgroup $\mathcal{U}$ (by [11, Corollary 8.34]), one has

$$
d_{1}(\mathcal{V}) \leqslant d_{1}(\mathcal{U})=d:=\text { dimension of } G
$$

for all closed subgroups $\mathcal{V}$ of $\mathcal{U}$. For $d_{2}(\mathcal{V})$ (i.e., the numbers of relations for a minimal set of topological generators of $\mathcal{V}$ ) the bound is provided by [11, Theorem 4.35] (see also [11, Chapter 4, Exercise 11]) again only in terms of the dimension and rank of $G$ (for example, if $\mathcal{U}$ is as above, then $d_{2}(\mathcal{U})=\frac{d(d-1)}{2}$ ). We put $\tilde{d}_{1}=\tilde{d}_{1}(G)$ (respectively $\tilde{d}_{2}=\tilde{d}_{2}(G)$ ) as the upper bound for all the $d_{1}(\mathcal{V})$ 's (respectively $d_{2}(\mathcal{V})$ 's) as $\mathcal{V}$ varies among the closed subgroups of any pro- $\ell$ Sylow of $G$.

Let $M_{\text {div }}$ be the maximal divisible subgroup of $M$ and consider the finite quotient $M / M_{\mathrm{div}}$. By [30, Chapter I, §4, Proposition 20] and the Corollaire right after it, the finite group $M / M_{\text {div }}$ admits a $\mathcal{V}$-composition series with quotients isomorphic to $\mathbb{Z} / \ell \mathbb{Z}$. Hence, working as in [13, Proposition 3.1], one immediately finds

$$
\left|H^{i}\left(\mathcal{V}, M / M_{\mathrm{div}}\right)\right| \leqslant\left|M / M_{\mathrm{div}}\right|^{d_{i}(\mathcal{V})} \leqslant\left|M / M_{\mathrm{div}}\right|^{\tilde{d}_{i}}
$$

for $i=1,2$.

Note that, if $M$ is finite (hence equal to $M / M_{\text {div }}$ ), we have already completed the proof of the finiteness of the $H^{i}(\mathcal{V}, M)$. 
Moreover, in this case the orders are also bounded independently of $\mathcal{V}$. To deal with the divisible part note that $M_{\mathrm{div}}[\ell]$ is finite (say of order $\ell^{\lambda}$, where $\lambda=\operatorname{corank}_{\mathbb{Z}_{\ell}} M$ ), thus, by what we have just proved,

$$
\left|H^{i}\left(\mathcal{V}, M_{\mathrm{div}}[\ell]\right)\right| \leqslant \ell^{\lambda \tilde{d}_{i}} \quad(i=1,2) .
$$

The cohomology of the exact sequence

$$
M_{\mathrm{div}}[\ell] \hookrightarrow M_{\mathrm{div}} \stackrel{\ell}{\rightarrow} M_{\mathrm{div}}
$$

yields surjective morphisms

$$
H^{i}\left(\mathcal{V}, M_{\mathrm{div}}[\ell]\right) \rightarrow H^{i}\left(\mathcal{V}, M_{\mathrm{div}}\right)[\ell] \quad(i=1,2),
$$

therefore the groups on the right have finite and bounded orders as well.

Hence the $H^{i}\left(\mathcal{V}, M_{\text {div }}\right)$ are cofinitely generated $\mathbb{Z}_{\ell}$-modules with coranks bounded by

$$
\tilde{d}_{i} \lambda=\tilde{d}_{i} \operatorname{corank}_{\mathbb{Z}_{\ell}} M
$$

(which is a bound for the whole $H^{i}(\mathcal{V}, M)$ since the $\mathbb{Z}_{\ell \text {-corank of the finite part }}$ is 0$)$.

Theorem 3.2. With the above notations, for every finite extension $F^{\prime}$ of $F$ contained in $K$, the kernels and cokernels of the maps

$$
a_{K / F^{\prime}}: \operatorname{Sel}_{A}\left(F^{\prime}\right)_{\ell} \rightarrow \operatorname{Sel}_{A}(K)_{\ell}^{\mathrm{Gal}\left(K / F^{\prime}\right)}
$$

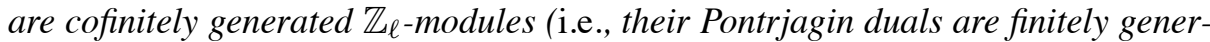
ated $\mathbb{Z}_{\ell}$-modules). If all primes in $S$ and all primes of bad reduction have decomposition groups open in $G$, then the coranks of kernels and cokernels are bounded independently of $F^{\prime}$. Moreover if $A\left[\ell^{\infty}\right](K)$ is finite, then such kernels and cokernels are of finite order.

Proof. We shall use the snake lemma for the diagram (2.1): hence, to prove the theorem, we are going to bound the kernels and cokernels of the maps $b_{K / F^{\prime}}$ and $c_{K / F^{\prime}}$

\subsection{The maps $b_{K / F^{\prime}}$}

From the Hochschild-Serre spectral sequence we have that

$\operatorname{Ker}\left(b_{K / F^{\prime}}\right) \simeq H^{1}\left(K / F^{\prime}, A\left[\ell^{\infty}\right](K)\right)$ and $\operatorname{Coker}\left(b_{K / F^{\prime}}\right) \subseteq H^{2}\left(K / F^{\prime}, A\left[\ell^{\infty}\right](K)\right)$.

We can apply Lemma 3.1 with $G=\operatorname{Gal}(K / F)$ and $M=A\left[\ell^{\infty}\right](K)$. Hence $\operatorname{Ker}\left(b_{K / F^{\prime}}\right)$ and $\operatorname{Coker}\left(b_{K / F^{\prime}}\right)$ are cofinitely generated $\mathbb{Z}_{\ell-\text { modules whose coranks }}$ are bounded by

$$
\tilde{d}_{1}(\operatorname{Gal}(K / F)) \operatorname{corank}_{\mathbb{Z}_{\ell}} A\left[\ell^{\infty}\right](K) \text { and } \tilde{d}_{2}(\operatorname{Gal}(K / F)) \operatorname{corank}_{\mathbb{Z}_{\ell}} A\left[\ell^{\infty}\right](K)
$$


respectively. Moreover, if $A\left[\ell^{\infty}\right](K)$ is finite, we have the bound

$$
\left|H^{i}\left(\mathcal{V}, A\left[\ell^{\infty}\right](K)\right)\right| \leqslant\left|A\left[\ell^{\infty}\right](K)\right|^{\tilde{d}_{i}(\operatorname{Gal}(K / F))}
$$

(for $i=1,2$ ).

\subsection{The maps $c_{K / F^{\prime}}$}

For every prime $v \in \Sigma_{F}$, let $v^{\prime}$ be a place of $F^{\prime}$ lying above $v$. Observe that

$$
\operatorname{Ker}\left(c_{K / F^{\prime}}\right) \hookrightarrow \prod_{v^{\prime} \in \Sigma_{F^{\prime}}} \bigcap_{\substack{w \in \Sigma_{K} \\ w \mid v^{\prime}}} \operatorname{Ker}\left(d_{w}\right)
$$

where

$$
d_{w}: H^{1}\left(F_{v^{\prime}}^{\prime}, A\left[\ell^{\infty}\right]\right) \rightarrow H^{1}\left(K_{w}, A\left[\ell^{\infty}\right]\right)
$$

and, by the Inf-Res sequence,

$$
\operatorname{Ker}\left(d_{w}\right)=H^{1}\left(K_{w} / F_{v^{\prime}}^{\prime}, A\left[\ell^{\infty}\right]\left(K_{w}\right)\right) .
$$

From the Kummer exact sequence one can write the following diagram

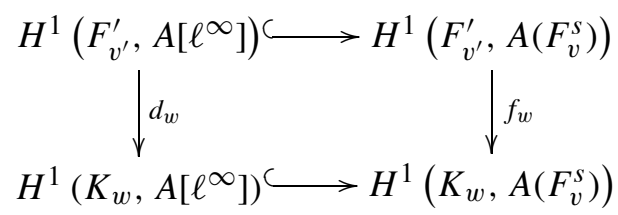

and deduce from it the inclusion

$$
\operatorname{Ker}\left(d_{w}\right) \hookrightarrow \operatorname{Ker}\left(f_{w}\right) \simeq H^{1}\left(K_{w} / F_{v^{\prime}}^{\prime}, A\left(K_{w}\right)\right) .
$$

Since this last group is obviously trivial if the prime $v^{\prime}$ splits completely we limit ourselves to the study of these local kernels for primes which are not totally split in $K / F^{\prime}$.

If $v$ is a prime of good reduction for $A$ and unramified in $K / F^{\prime}$, then [21, Chapter I, Proposition 3.8] yields

$$
H^{1}\left(\left(F_{v^{\prime}}^{\prime}\right)^{\mathrm{unr}} / F_{v^{\prime}}^{\prime}, A\left(\left(F_{v^{\prime}}^{\prime}\right)^{\mathrm{unr}}\right)\right)=0 .
$$

Via the inflation map one immediately gets $H^{1}\left(K_{w} / F_{v^{\prime}}^{\prime}, A\left(K_{w}\right)\right)=0$.

Thus we are left with

$$
\operatorname{Ker}\left(c_{K / F^{\prime}}\right) \hookrightarrow \prod_{\substack{v^{\prime} \in \Sigma_{F^{\prime}} \\
v^{\prime} \mid v \in S^{*}}} \bigcap_{\begin{array}{c}
w \in \Sigma_{K} \\
w \mid v^{\prime}
\end{array}} \operatorname{Ker}\left(d_{w}\right)
$$


where $S^{*}$ is the finite set composed by:

- all primes in $S$;

- all primes not in $S$ and of bad reduction for $A$.

To find bounds for these primes we shall use Tate's theorems on (local) duality and (local) Euler-Poincaré characteristic.

By [22, Theorem 7.1.8], the group $H^{1}\left(F_{v^{\prime}}^{\prime}, A\left[\ell^{\infty}\right]\right)$ is finite and we can bound its order using the Euler characteristic

$$
\chi\left(F_{v^{\prime}}^{\prime}, A\left[\ell^{\infty}\right]\right):=\frac{\left|H^{0}\left(F_{v^{\prime}}^{\prime}, A\left[\ell^{\infty}\right]\right)\right|\left|H^{2}\left(F_{v^{\prime}}^{\prime}, A\left[\ell^{\infty}\right]\right)\right|}{\left|H^{1}\left(F_{v^{\prime}}^{\prime}, A\left[\ell^{\infty}\right]\right)\right|} .
$$

From the pairing on cohomology induced by the Weil pairing (see, for example, [21, Chapter I, Remark 3.5]), the group $H^{2}\left(F_{v^{\prime}}^{\prime}, A\left[\ell^{\infty}\right]\right)$ is the (Pontrjagin) dual of $H^{0}\left(F_{v^{\prime}}^{\prime}, B\left[\ell^{\infty}\right]\right)$, so all the orders in the formula are finite. Moreover, by [22, Theorem 7.3.1], $\chi\left(F_{v^{\prime}}^{\prime}, A\left[\ell^{\infty}\right]\right)=1^{1}$, therefore

$$
\left|H^{1}\left(F_{v^{\prime}}^{\prime}, A\left[\ell^{\infty}\right]\right)\right|=\left|A\left[\ell^{\infty}\right]\left(F_{v^{\prime}}^{\prime}\right)\right|\left|B\left[\ell^{\infty}\right]\left(F_{v^{\prime}}^{\prime}\right)\right| .
$$

The inflation map

$$
H^{1}\left(K_{w} / F_{v^{\prime}}^{\prime}, A\left[\ell^{\infty}\right]\left(K_{w}\right)\right) \hookrightarrow H^{1}\left(F_{v^{\prime}}^{\prime}, A\left[\ell^{\infty}\right]\right)
$$

provides again the finiteness of the kernels (but note that here, in general, the orders are not bounded).

\section{Remark 3.3.}

1. In the previous theorem (and in all control theorems which will follow, i.e., Theorems 3.7, 4.8, 5.3 and 6.4) we do not require any assumption on the reduction of $A$ outside $S$. Hypotheses of that kind were used, for example, in [23, Theorem 1.9].

2. Note that the local kernels are always finite, the additional hypothesis on the finiteness of $A\left[\ell^{\infty}\right](K)$ was only used to bound the orders of $\operatorname{Ker}\left(b_{K / F^{\prime}}\right)$ and $\operatorname{Coker}\left(b_{K / F^{\prime}}\right)$.

3. Most of the bounds are independent of $F^{\prime}$ (in particular the ones for $\operatorname{Ker}\left(a_{K / F^{\prime}}\right)$ ), but to get uniform bounds for $\operatorname{Coker}\left(a_{K / F^{\prime}}\right)$ one also needs to bound the number of nontrivial groups appearing in the product which contains $\operatorname{Ker}\left(c_{K / F^{\prime}}\right)$. In particular one needs finitely many nontrivial $\operatorname{Ker}\left(d_{w}\right)$ 's and the only way to get

1 The statement is for finite modules (i.e., it's true for all the modules $A\left[\ell^{n}\right]$ for any $n$ ), but limits are allowed here since the numerator stabilizes, i.e., there is an $n$ such that

$$
\left|A\left[\ell^{m}\right]\left(F_{v^{\prime}}^{\prime}\right)\right|\left|B\left[\ell^{m}\right]\left(F_{v^{\prime}}^{\prime}\right)\right|=\left|A\left[\ell^{n}\right]\left(F_{v^{\prime}}^{\prime}\right)\right|\left|B\left[\ell^{n}\right]\left(F_{v^{\prime}}^{\prime}\right)\right|
$$

for all $m \geqslant n$. 
this is assuming that the decomposition groups are open. Note that if there is at least one unramified prime of bad reduction such hypothesis on its decomposition group immediately yields that $G$ contains a subgroup isomorphic to $\mathbb{Z}_{\ell}$ with finite index.

We can be a bit more precise with the local bounds for the unramified primes of bad reduction thanks to the following

Proposition 3.4. If $v$ is an unramified prime of bad reduction (with nontrivial decomposition group) and $v^{\prime} \mid v$, then $H^{1}\left(K_{w} / F_{v^{\prime}}^{\prime}, A\left[\ell^{\infty}\right]\left(K_{w}\right)\right)$ is finite and its order is bounded independently of $F^{\prime}$.

Proof. The $\ell$-part of the Galois group $\operatorname{Gal}\left(K_{w} / F_{v^{\prime}}^{\prime}\right)$ is a finite cyclic $\ell$-group or it is isomorphic to $\mathbb{Z}_{\ell}$. Moreover

$$
A\left[\ell^{\infty}\right]\left(K_{w}\right)^{\operatorname{Gal}\left(K_{w} / F_{v^{\prime}}^{\prime}\right)}=A\left[\ell^{\infty}\right]\left(F_{v^{\prime}}^{\prime}\right)
$$

is finite, so, in the $\mathbb{Z}_{\ell}$ case (i.e., when $K_{w}$ contains $\left(F_{v^{\prime}}^{\prime}\right)^{\ell, u n r}$ the unramified $\mathbb{Z}_{\ell^{-}}$ extension of $\left.F_{v^{\prime}}^{\prime}\right)$, we can apply [5, Remark 3.5] to get

$$
\left|H^{1}\left(K_{w} / F_{v^{\prime}}^{\prime}, A\left[\ell^{\infty}\right]\left(K_{w}\right)\right)\right| \leqslant\left|A\left[\ell^{\infty}\right]\left(K_{w}\right) / A\left[\ell^{\infty}\right]\left(K_{w}\right)_{\operatorname{div}}\right| .
$$

A similar bound (independent from $F^{\prime}$ ) holds for the finite case as well. One just uses the inflation map to the group $H^{1}\left(K_{w}\left(F_{v^{\prime}}^{\prime}\right)^{\ell, u n r} / F_{v^{\prime}}^{\prime}, A\left[\ell^{\infty}\right]\left(K_{w}\left(F_{v^{\prime}}^{\prime}\right)^{\ell, u n r}\right)\right)$ which has order bounded by $\left|A\left[\ell^{\infty}\right]\left(K_{w}\left(F_{v}\right)^{\ell, u n r}\right) / A\left[\ell^{\infty}\right]\left(K_{w}\left(F_{v}\right)^{\ell, u n r}\right)_{\operatorname{div}}\right|$.

We summarize the given bounds with the following

Corollary 3.5. In the setting of Theorem 3.2 assume that all primes in $S$ and all primes of bad reduction have decomposition groups open in $G$, then one has:

1. $\operatorname{corank}_{\mathbb{Z}_{\ell}} \operatorname{Ker}\left(a_{K / F^{\prime}}\right) \leqslant \tilde{d}_{1} \operatorname{corank}_{\mathbb{Z}_{\ell}} A\left[\ell^{\infty}\right](K)$ and $\operatorname{corank}_{\mathbb{Z}_{\ell}} \operatorname{Coker}\left(a_{K / F^{\prime}}\right) \leqslant \tilde{d}_{2} \operatorname{corank}_{\mathbb{Z}_{\ell}} A\left[\ell^{\infty}\right](K)$;

2. if $A\left[\ell^{\infty}\right](K)$ is finite, then $\left|\operatorname{Ker}\left(a_{K / F^{\prime}}\right)\right| \leqslant\left|A\left[\ell^{\infty}\right](K)\right|^{\tilde{d}_{1}}$ and

$$
\left|\operatorname{Coker}\left(a_{K / F^{\prime}}\right)\right| \leqslant\left|A\left[\ell^{\infty}\right](K)\right|^{\tilde{d}_{2}} \prod_{v^{\prime} \mid v \in S^{*}-S} \alpha_{v} \prod_{v^{\prime} \mid v \in S} \beta_{v^{\prime}}
$$

(where

$$
\alpha_{v}=\left|A\left[\ell^{\infty}\right]\left(K_{w}\left(F_{v}\right)^{\ell, u n r}\right) / A\left[\ell^{\infty}\right]\left(K_{w}\left(F_{v}\right)^{\ell, u n r}\right)_{\operatorname{div}}\right|
$$

and

$$
\left.\beta_{v^{\prime}}=\left|A\left[\ell^{\infty}\right]\left(F_{v^{\prime}}^{\prime}\right)\right|\left|B\left[\ell^{\infty}\right]\left(F_{v^{\prime}}^{\prime}\right)\right|\right)
$$


3. if, for all primes $v \in S, A\left[\ell^{\infty}\right]\left(K_{w}\right)$ is finite for all primes $w\left|v^{\prime}\right| v$ (hence $A\left[\ell^{\infty}\right](K)$ is finite as well), then $\left|\operatorname{Ker}\left(a_{K / F^{\prime}}\right)\right| \leqslant\left|A\left[\ell^{\infty}\right](K)\right|^{\tilde{d}_{1}}$ and

$$
\left|\operatorname{Coker}\left(a_{K / F^{\prime}}\right)\right| \leqslant\left|A\left[\ell^{\infty}\right](K)\right|^{\tilde{d}_{2}} \prod_{v^{\prime} \mid v \in S^{*}-S} \alpha_{v} \prod_{v^{\prime} \mid v \in S}\left|A\left[\ell^{\infty}\right]\left(K_{w}\right)\right|\left|B\left[\ell^{\infty}\right]\left(K_{w}\right)\right| .
$$

The bounds in 1 are independent of $F^{\prime}$ (while the values appearing in $\mathbf{3}$ do not depend on $F^{\prime}$ but the number of the factors in the product does).

\subsection{The case $K=F\left(A\left[\ell^{\infty}\right]\right)$}

The finiteness of $A\left[\ell^{\infty}\right](K)$ is not a necessary condition to get finite kernels and cokernels in the control theorem. An important example is provided by the extension $K=F\left(A\left[\ell^{\infty}\right]\right)$ (this extensions has been studied in details in [27] in the case $A=E$, an elliptic curve). Fix a basis of $A\left[\ell^{\infty}\right]$ and consider the continuous Galois representation

$$
\rho: \operatorname{Gal}\left(F^{s} / F\right) \rightarrow G L_{2 g}\left(\mathbb{Z}_{\ell}\right)
$$

provided by the action of $\operatorname{Gal}\left(F^{s} / F\right)$ on the chosen basis. Since $\operatorname{Ker}(\rho)$ is given by the automorphisms which fix $A\left[\ell^{\infty}\right]$, one gets an isomorphism

$$
\operatorname{Gal}(K / F) \simeq \rho\left(\operatorname{Gal}\left(F^{s} / F\right)\right)
$$

and, consequently, an embedding

$$
\operatorname{Gal}(K / F) \hookrightarrow G L_{2 g}\left(\mathbb{Z}_{\ell}\right)
$$

Since $\operatorname{Gal}\left(F^{s} / F\right)$ is compact, its image under $\rho$ must be a compact subgroup of $G L_{2 g}\left(\mathbb{Z}_{\ell}\right)$. As the latter is Hausdorff, $\rho\left(\operatorname{Gal}\left(F^{s} / F\right)\right)$ is closed and so it is an $\ell$-adic Lie group.

Remark 3.6. When $A$ has genus 1 (i.e., it is an elliptic curve), a theorem of Igusa (analogous to Serre's open image theorem) gives a more precise description of $\operatorname{Gal}(K / F)$ (for a precise statement and a proof see [6] and the references there). For a general abelian variety such open image statements are not known: some results in this direction for abelian varieties of "Hall type" can be found in [14] and [1].

Theorem 3.7. Let $K=F\left(A\left[\ell^{\infty}\right]\right)$, then the kernels and cokernels of the maps

$$
a_{K / F^{\prime}}: \operatorname{Sel}_{A}\left(F^{\prime}\right)_{\ell} \rightarrow \operatorname{Sel}_{A}(K)_{\ell}^{\mathrm{Gal}\left(K / F^{\prime}\right)}
$$

are finite.

Proof. Observe that thanks to [31, Corollary 2 (b), page 497] only primes of bad reduction for $A$ are ramified in the extension $F\left(A\left[\ell^{\infty}\right]\right) / F$; so, in this case, the set $S$ is obviously finite and there are no unramified primes of bad reduction. 
The bounds for $\operatorname{Ker}\left(c_{K / F^{\prime}}\right)$ are already in Theorem 3.2, so we only need to provide bounds for $\operatorname{Ker}\left(b_{K / F^{\prime}}\right)$ and $\operatorname{Coker}\left(b_{K / F^{\prime}}\right)$, i.e., for $H^{1}\left(K / F^{\prime}, A\left[\ell^{\infty}\right]\right)$ and $H^{2}\left(K / F^{\prime}, A\left[\ell^{\infty}\right]\right)$ (note that here $A\left[\ell^{\infty}\right](K)=A\left[\ell^{\infty}\right]$ ).

Consider the Tate module

$$
T_{\ell}(A):=\lim _{n} A\left[\ell^{n}\right] \quad \text { and put } \quad V_{\ell}(A):=T_{\ell}(A) \otimes_{\mathbb{Z}_{\ell}} \mathbb{Q}_{\ell}
$$

then one has an exact sequence (of Galois modules)

$$
T_{\ell}(A) \hookrightarrow V_{\ell}(A) \rightarrow A\left[\ell^{\infty}\right] .
$$

By [29, Théorème 2] and the subsequent Corollaire (which hold in our setting as well, as noted in the Remarques following the Corollaire), one has $H^{i}\left(K / F^{\prime}, V_{\ell}(A)\right)=0$ for any $i \geqslant 0$, moreover the $H^{i}\left(K / F^{\prime}, T_{\ell}(A)\right)$ are all finite groups. Hence we get isomorphism

$$
H^{i}\left(K / F^{\prime}, T_{\ell}(A)\right) \simeq H^{i-1}\left(K / F^{\prime}, A\left[\ell^{\infty}\right]\right) \quad \text { for any } i \geqslant 1
$$

which provide the finiteness of $\operatorname{Ker}\left(b_{K / F^{\prime}}\right)$ and $\operatorname{Coker}\left(b_{K / F^{\prime}}\right)$.

\section{4. $\Lambda$-modules for $\ell \neq p$}

In this section we assume that our Galois group $G$ (still an $\ell$-adic Lie group) has no elements of order $\ell$ and write $\Lambda(G)$ for the associated Iwasawa algebra. First we describe the structure of $\operatorname{Sel}_{A}(K)_{\ell}^{\vee}$ as a $\Lambda(G)$-module, showing that it is a finitely generated (sometimes torsion) $\Lambda(G)$-module. Then, assuming that $G$ contains a subgroup $H$ such that $G / H \simeq \mathbb{Z}_{\ell}$, we will show that $\operatorname{Sel}_{A}(K)_{\ell}^{\vee}$ is finitely generated as a $\Lambda(H)$-module as well. For the latter we shall need a slightly modified version of Theorem 3.2. As usual the main tool for the proof (along with the control theorem) is the following generalization of Nakayama's Lemma.

Theorem 4.1. Let $G$ be a topologically finitely generated, powerful and pro- $\ell$ group and I any proper ideal. Let $M$ be a compact $\Lambda(G)$-module, then:

1. if $M / I M$ is finitely generated as a $\Lambda(G) / I$-module then $M$ is finitely generated as a $\Lambda(G)$-module;

2. if $G$ is soluble uniform and $M / I_{G} M$ is finite then $M$ is a torsion $\Lambda(G)$-module (where $I_{G}=\operatorname{Ker}\left(\Lambda(G) \rightarrow \mathbb{Z}_{\ell}\right)$ is the augmentation ideal of $\left.\Lambda(G)\right)$.

Proof. See the main results of [2].

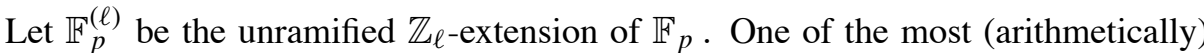
interesting example is provided by extensions $K / F$ containing $\mathbb{F}_{p}^{(\ell)} F$, where one can take $H=\operatorname{Gal}\left(K / \mathbb{F}_{p}^{(\ell)} F\right)$. This can be considered as a very general setting thanks to the following lemma (which was brought to our attention by David Burns). 
Lemma 4.2. Let $K / F$ be an $\ell$-adic Lie extension with $\ell \neq 2, p$. Then there exist a field $K^{\prime} \supseteq K$ such that

1. $K^{\prime}$ contains $\mathbb{F}_{p}^{(\ell)} F$;

2. $K^{\prime} / F$ is unramified outside a finite set of places;

3. $\mathrm{Gal}\left(K^{\prime} / F\right)$ is a compact $\ell$-adic Lie group without elements of order $\ell$.

Proof. Since $\ell \neq p$ the proof is the same of [8, Lemma 6.1] (where the statement is for number fields). One basically considers the field $K\left(\boldsymbol{\mu}_{\ell^{\infty}}\right)$ (which obviously contains $\left.\mathbb{F}_{p}^{(\ell)} F\right)$, where $\mu_{\ell^{\infty}}$ is the set of all $\ell$-power roots of unity, and then cuts out elements of order $\ell$ in $\operatorname{Gal}\left(K\left(\boldsymbol{\mu}_{\ell^{\infty}}\right) / F\left(\boldsymbol{\mu}_{\ell^{\infty}}\right)\right)$ using Kummer theory to describe generators for subextensions of degree $\ell$.

\subsection{Structure of $\operatorname{Sel}_{A}(K)_{\ell}^{\vee}$ as $\Lambda(G)$-module}

We are now ready to prove the following:

Theorem 4.3. In the setting of Theorem 3.2, $\operatorname{Sel}_{A}(K)_{\ell}^{\vee}$ is a finitely generated $\Lambda(G)$-module.

Proof. Consider any open, powerful and pro- $\ell$ subgroup $G^{\prime}$ of $G$. Since $\Lambda(G)$ is finitely generated over $\Lambda\left(G^{\prime}\right)$ it is obvious that $\operatorname{Sel}_{A}(K)_{\ell}^{\vee}$ is finitely generated over $\Lambda(G)$ if and only if it is finitely generated also over $\Lambda\left(G^{\prime}\right)$. So we are going to prove the statement for such $G^{\prime}$.

Consider the exact sequence

$$
0 \rightarrow \operatorname{Coker}\left(a_{K / F^{\prime}}\right)^{\vee} \rightarrow\left(\operatorname{Sel}_{A}(K)_{\ell}^{G^{\prime}}\right)^{\vee} \rightarrow \operatorname{Sel}_{A}\left(F^{\prime}\right)_{\ell}^{\vee} \rightarrow \operatorname{Ker}\left(a_{K / F^{\prime}}\right)^{\vee} \rightarrow 0
$$

where $F^{\prime}$ is the fixed field of $G^{\prime}$. We know from Theorem 3.2 that $\operatorname{Coker}\left(a_{K / F^{\prime}}\right)^{\vee}$

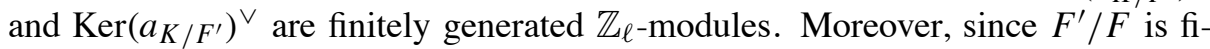
nite, $\operatorname{Sel}_{A}\left(F^{\prime}\right)_{\ell}^{\vee}$ is a finitely generated $\mathbb{Z}_{\ell}$-module ( [21, III.8 and III.9]). Hence $\left(\operatorname{Sel}_{A}(K)_{\ell}^{G^{\prime}}\right)^{\vee}$ is a finitely generated $\mathbb{Z}_{\ell}$-module thanks to the exactness of the sequence (4.1). Since $\left(\operatorname{Sel}_{A}(K)_{\ell}^{G^{\prime}}\right)^{\vee}$ is isomorphic to $\operatorname{Sel}_{A}(K)_{\ell}^{\vee} / I_{G^{\prime}} \operatorname{Sel}_{A}(K)_{\ell}^{\vee}$, where $I_{G^{\prime}}$ is the augmentation ideal, our claim follows from Theorem 4.1.

Remark 4.4. Note that in the above proof we do not need any hypothesis on the elements of $G$ of order $\ell$ : it works in general for any compact $\ell$-adic Lie group $G$. That additional hypothesis is necessary only to prove that $\operatorname{Sel}_{A}(K)_{\ell}^{\vee}$ is a torsion module, because we need to avoid zero divisors.

Theorem 4.5. Suppose that there exists an open uniform, pro- $\ell$ and soluble subgroup $G^{\prime}$ of $G$, with fixed field $F^{\prime}$. Assume that $A\left[\ell^{\infty}\right](K)$ and $\operatorname{Sel}_{A}\left(F^{\prime}\right)_{\ell}^{\vee}$ are finite. Then $\operatorname{Sel}_{A}(K)_{\ell}^{\vee}$ is a torsion $\Lambda\left(G^{\prime}\right)$-module.

Proof. Just use Theorems 3.2 and 4.1. 
Remark 4.6. The hypothesis on the existence of the soluble subgroup $G^{\prime}$ is necessary. Indeed, when $G^{\prime}$ is not soluble it is possible to find a non torsion ideal $J$ of $\Lambda\left(G^{\prime}\right)$ such that $J / I_{G^{\prime}} J$ is finite (see [2, page 228]). However, we observe that when $G$ is finitely generated (not only "topologically" finitely generated) such an open soluble subgroup $G^{\prime}$ always exists (see [18]).

In the context of non-commutative Iwasawa algebras the right definition of torsion module ([36, Definition 2.6]) can be stated in the following way: a finitely generated $\Lambda(G)$-module $M$ is a $\Lambda(G)$-torsion module if and only if $M$ is a $\Lambda\left(G^{\prime}\right)$-torsion module (classical meaning) for some open pro- $\ell$ subgroup $G^{\prime} \subseteq G$ such that $\Lambda\left(G^{\prime}\right)$ is integral. So Theorem 4.5 immediately yields.

Corollary 4.7. Let $G$ be without elements of order $\ell$ and suppose that there exists an open, uniform, pro- $\ell$ and soluble subgroup $G^{\prime}$ of $G$. If $A\left[\ell^{\infty}\right](K)$ and $\operatorname{Sel}_{A}\left(F^{\prime}\right)_{\ell}^{\vee}$ (where $F^{\prime}$ is the fixed field of $G^{\prime}$ ) are finite, then $\operatorname{Sel}_{A}(K)_{\ell}^{\vee}$ is a torsion $\Lambda(G)$ module.

\subsection{Structure of $\operatorname{Sel}_{A}(K)_{\ell}^{\vee}$ as $\Lambda(H)$-module}

Assume that $G$ contains a closed normal subgroup $H$ such that $G / H=\Gamma \simeq \mathbb{Z}_{\ell}$. We are going to prove that $\operatorname{Sel}_{A}(K)_{\ell}^{\vee}$ is a finitely generated $\Lambda(H)$-module under some mild conditions, as predicted by Conjecture 5.1 of [9]. First note that, letting $\mathbb{F}_{p}^{(\ell)}$ be the unique $\mathbb{Z}_{\ell}$-extension of $\mathbb{F}_{p}$, by [5, Proposition 4.3], one has $K^{\prime}:=$ $K^{H}=\mathbb{F}_{p}^{(\ell)} F$. Hence all primes of $F$ are unramified in $K^{\prime}$ and none of them is totally split.

As mentioned before we need to prove a slightly modified version of the Control Theorem. We will work with the following diagram

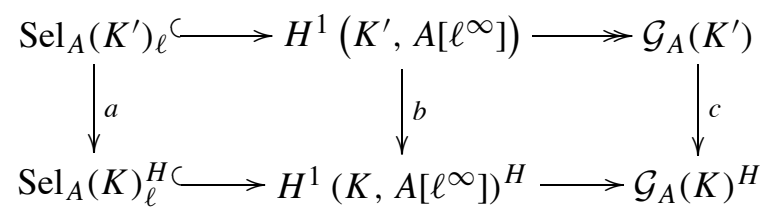

similar to the diagram (2.1) except for the "infinite level" of the upper row, and we will again apply the snake lemma.

Theorem 4.8. With the above notations the kernel and cokernel of the map

$$
a: \operatorname{Sel}_{A}\left(K^{\prime}\right)_{\ell} \rightarrow \operatorname{Sel}_{A}(K)_{\ell}^{H}
$$

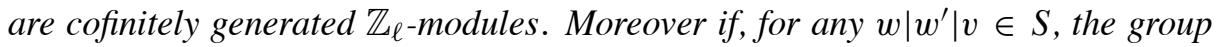
$A\left[\ell^{\infty}\right]\left(K_{w}\right)$ is finite, then $\operatorname{Ker}(a)$ and $\operatorname{Coker}(a)$ are finite.

Proof. As usual we are going to work on kernels and cokernels of the maps $b$ and $c$ in diagram (4.2). 


\subsection{The map $b$}

From the Hochschild-Serre spectral sequence we have that

$$
\operatorname{Ker}(b) \simeq H^{1}\left(K / K^{\prime}, A\left[\ell^{\infty}\right](K)\right) \quad \text { and } \quad \operatorname{Coker}(b) \subseteq H^{2}\left(K / K^{\prime}, A\left[\ell^{\infty}\right](K)\right) .
$$

One simply observes that $\mathrm{Gal}\left(K / K^{\prime}\right)$ is still an $\ell$-adic Lie group and then applies Lemma 3.1. Hence $\operatorname{Ker}(b)$ and $\operatorname{Coker}(b)$ are cofinitely generated $\mathbb{Z}_{\ell}$-modules and are finite if $A\left[\ell^{\infty}\right](K)$ is finite.

\subsection{The map $c$}

For every prime $v \in \Sigma_{F}$ let $w^{\prime}$ be a place of $K^{\prime}$ lying above $v$. As in Section 3.2, from the Inf-Res sequence, one gets

$$
\operatorname{Ker}\left(d_{w}\right)=H^{1}\left(K_{w} / K_{w^{\prime}}^{\prime}, A\left[\ell^{\infty}\right]\left(K_{w}\right)\right) .
$$

Since $\operatorname{Gal}\left(K_{w} / K_{w^{\prime}}^{\prime}\right)$ is an $\ell$-adic Lie group, every $\operatorname{Ker}\left(d_{w}\right)$ is a cofinitely generated $\mathbb{Z}_{\ell}$-module. The result for $\operatorname{Ker}(c)$ will follow from $\operatorname{Ker}\left(d_{w}\right)=0$ for all but finitely many primes. As before the Kummer sequence provides the following diagram

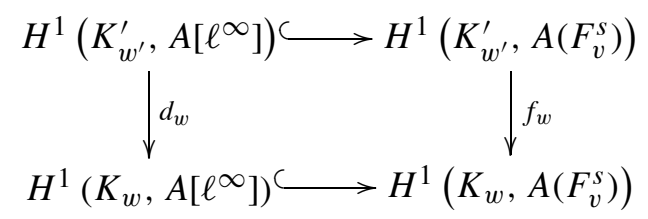

and, from it, one has the inclusion

$$
\operatorname{Ker}\left(d_{w}\right) \hookrightarrow \operatorname{Ker}\left(f_{w}\right) \simeq H^{1}\left(K_{w} / K_{w^{\prime}}^{\prime}, A\left(K_{w}\right)\right)
$$

or, more precisely, the isomorphism

$$
\operatorname{Ker}\left(d_{w}\right) \simeq H^{1}\left(K_{w} / K_{w^{\prime}}^{\prime}, A\left(K_{w}\right)\right)\left[\ell^{\infty}\right] .
$$

Hence we are not going to consider places which are totally split in $K / K^{\prime}$, because they obviously provide $\operatorname{Ker}\left(d_{w}\right)=0$.

\subsubsection{Unramified primes}

Let $v$ be unramified in $K / F$, then, since $\operatorname{Gal}\left(K_{w^{\prime}}^{\prime} / F_{v}\right) \simeq \mathbb{Z}_{\ell}, K_{w^{\prime}}^{\prime}$ is the maximal unramified pro- $\ell$-extension of $F_{v}$ and the $\ell$-part of $\operatorname{Gal}\left(K_{w} / K_{w^{\prime}}^{\prime}\right)$ is trivial. Therefore the $\ell$-part of the (torsion) module $H^{1}\left(K_{w} / K_{w^{\prime}}^{\prime}, A\left(K_{w}\right)\right)$ is trivial as well.

Because of the splitting of primes in $K^{\prime}$, we are already left with finitely many places. So $\operatorname{Ker}(c)$ is a cofinitely generated $\mathbb{Z}_{\ell}$-module and the first statement on $\operatorname{Ker}(a)$ and $\operatorname{Coker}(a)$ being cofinitely generated over $\mathbb{Z}_{\ell}$ is proved. 


\subsubsection{Ramified primes}

Let $v \in S$ and recall that $K_{w^{\prime}}^{\prime}$ is not a local field anymore. If for any $w\left|w^{\prime}\right| v$ the group $A\left[\ell^{\infty}\right]\left(K_{w}\right)$ is finite, then, by Lemma 3.1, $H^{1}\left(K_{w} / K_{w^{\prime}}^{\prime}, A\left[\ell^{\infty}\right]\left(K_{w}\right)\right)$ is finite.

Corollary 4.9. In the setting of Theorem 4.8, one has

1. if $\operatorname{Sel}_{A}\left(K^{\prime}\right)_{\ell}$ is a cofinitely generated $\mathbb{Z}_{\ell}$-module, then $\operatorname{Sel}_{A}(K)_{\ell}^{\vee}$ is finitely generated over $\Lambda(H)$;

2. suppose that there exists an open, soluble, uniform and pro- $\ell$ subgroup $H^{\prime}$ of $H$; if the groups $\operatorname{Sel}_{A}\left(K^{H^{\prime}}\right) \ell$ and $A\left[\ell^{\infty}\right]\left(K_{w}\right)$, for any $w\left|w^{\prime}\right| v \in S$, are finite, then $\operatorname{Sel}_{A}(K)_{\ell}^{\vee}$ is a torsion $\Lambda(H)$-module.

Proof. The arguments are the same we used for the analogous results for $\Lambda(G)$ modules.

Remark 4.10. To get $\operatorname{Sel}_{A}(K)_{\ell}^{\vee}$ finitely generated over $\Lambda(G)$ we only need to assume that finitely many primes of $F$ are ramified in the Lie extension $K / F$. More-

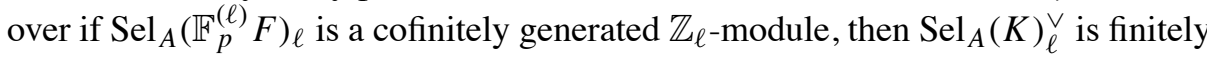
generated also over $\Lambda(H)$. Since $H$ has infinite index in $G$, being finitely generated over $\Lambda(G)$ and $\Lambda(H)$ implies that $\operatorname{Sel}_{A}(K)_{\ell}^{\vee}$ is $\Lambda(G)$-torsion (assuming that $G$ does not contain any element of order $\ell$ ). So this is another way to obtain torsion $\Lambda(G)$-modules without assuming the finiteness of $A\left[\ell^{\infty}\right](K)$ (which we needed in Corollary 4.7 and is obviously false for $\left.K=F\left(A\left[\ell^{\infty}\right]\right)\right)$. Moreover, as mentioned in the introduction, proving the finitely generated condition over $\Lambda(G)$ and $\Lambda(H)$ (as done also for all the cases included in Corollary 4.9) yields that $\operatorname{Sel}_{A}(K)_{\ell}^{\vee}$ is in the category $\mathfrak{M}_{H}(G)$, i.e., allows us to define a characteristic element for $\operatorname{Sel}_{A}(K)_{\ell}^{\vee}$ (without the $\Lambda(H)$-module structure, proving that a module is $\Lambda(G)$-torsion is not enough in the non-commutative case).

Example 4.11. Take $K=F\left(A\left[\ell^{\infty}\right]\right)$ as in Section 3.3. This kind of extension realizes naturally most of our assumptions. First of all, from [31], $S$ is just the set of places of bad reduction for $A$ and it is obviously finite (moreover $S=S^{*}$ in the notations of Section 3.2). As a consequence, by Theorem 4.3 we get $\operatorname{Sel}_{A}(K)_{\ell}^{\vee}$ always finitely generated over $\Lambda(G)$. Then, since $\operatorname{Gal}(K / F)$ embeds in $G L_{2 g}\left(\mathbb{Z}_{\ell}\right)$, it is easy to see that for $\ell>2 g+1$ the Galois group contains no elements of order $\ell$ so it makes sense to look for torsion modules. By the Weil-pairing we can take $H$ such that $K^{\prime} / F$ is the unramified $\mathbb{Z}_{\ell}$-extension of the constant field of $F$. Because of our choice of $H$, primes in $K^{\prime}$ above those in $S$ are finitely many. So, thanks to Theorem 4.8 if $\operatorname{Sel}_{A}\left(K^{\prime}\right)_{\ell}^{\vee}$ is finitely generated over $\mathbb{Z}_{\ell}$, then $\operatorname{Sel}_{A}(K)_{\ell}^{\vee}$ is also finitely generated over $\Lambda(H)$ (hence $\Lambda(G)$-torsion).

One can provide examples of Selmer groups $\operatorname{Sel}_{A}\left(K^{\prime}\right)_{\ell}$ cofinitely generated over $\mathbb{Z}_{\ell}$ in the work of Pacheco ([24, Proposition 3.6], which generalizes to abelian varieties the analogous statement of Ellenberg in [12, Proposition 2.5] for elliptic curves). For more details on the application of Ellenberg's results to the non- 
commutative setting of $F\left(A\left[\ell^{\infty}\right]\right) / F$ (like computations of coranks and Euler characteristic of Selmer groups) see Sechi's (unpublished) PhD thesis [27].

\section{Control theorem for $\ell=p$}

In order to work with the $p$-torsion part we need to use flat cohomology as mentioned in Section 2. We will work with diagram (2.1) but, as we will see, the kernels and cokernels appearing in the snake lemma sequence will still be described in terms of Galois cohomology groups. The main difference is provided by the fact that the images of the local Kummer maps will be nontrivial.

To handle the local kernels we shall need the following:

Lemma 5.1. Let $E$ be a local function field and let $L / E$ be an (infinite) $p$-adic Lie extension. Let $A$ be an abelian variety defined over $E$ with good ordinary reduction. Let I be the inertia group in $\mathrm{Gal}(L / E)$ and assume it is nontrivial. Then $H^{1}(L / E, A(L))$ (Galois cohomology group) is a cofinitely generated $\mathbb{Z}_{p}$-module. Moreover, if I has finite index (i.e., it is open) in $\operatorname{Gal}(L / E)$, then $H^{1}(L / E, A(L))$ is finite.

Proof. Let $\widehat{A}$ (respectively $\bar{A}$ ) be the formal group associated to $A$ (respectively the reduction of $A$ at the prime of $E$ ). Because of good reduction the natural map $A\left(E^{\prime}\right) \rightarrow \bar{A}\left(\mathbb{F}_{E^{\prime}}\right)$ is surjective for any extension $E^{\prime} / E$. Hence the sequence

$$
\widehat{A}\left(\mathcal{O}_{L}\right) \hookrightarrow A(L) \rightarrow \bar{A}\left(\mathbb{F}_{L}\right)
$$

is exact. Taking $\operatorname{Gal}(L / E)$ cohomology (and recalling that $A(E) \rightarrow \bar{A}\left(\mathbb{F}_{E}\right)$ is surjective), one gets

$$
H^{1}\left(L / E, \widehat{A}\left(\mathcal{O}_{L}\right)\right) \hookrightarrow H^{1}(L / E, A(L)) \rightarrow H^{1}\left(L / E, \bar{A}\left(\mathbb{F}_{L}\right)\right)
$$

and we will focus on the right and left terms of this sequence from now on.

By [34, Theorem 2 (a)], one has an isomorphism

$$
H^{1}\left(E, \widehat{A}\left(\mathcal{O}_{\bar{E}}\right)\right) \simeq \operatorname{Hom}\left(\bar{B}\left[p^{\infty}\right]\left(\mathbb{F}_{E}\right), \mathbb{Q}_{p} / \mathbb{Z}_{p}\right)
$$

(the statement of the theorem requires a $\mathbb{Z}_{p}^{d}$-extension but part (a) holds independently of that). Therefore the inflation map provides an inclusion

$$
H^{1}\left(L / E, \widehat{A}\left(\mathcal{O}_{L}\right)\right) \hookrightarrow \operatorname{Hom}\left(\bar{B}\left[p^{\infty}\right]\left(\mathbb{F}_{E}\right), \mathbb{Q}_{p} / \mathbb{Z}_{p}\right)
$$

and, since $\bar{A}$ and $\bar{B}$ are isogenous, the term on the right is finite of order $\left|\bar{B}\left[p^{\infty}\right]\left(\mathbb{F}_{E}\right)\right|=\left|\bar{A}\left[p^{\infty}\right]\left(\mathbb{F}_{E}\right)\right|$. 
For $H^{1}\left(L / E, \bar{A}\left(\mathbb{F}_{L}\right)\right)$ we consider two cases:

\subsection{Case 1: $I$ is open in $\operatorname{Gal}(L / E)$.}

Just considering $p$-parts we can assume that $I$ is open in a $p$-Sylow $P_{p}$ of $\operatorname{Gal}(L / E)$ and we let $L^{I}$ be its fixed field. Let $\overline{P_{p}}:=\operatorname{Gal}\left(L^{I} / E\right)$ (a finite group) and note that, since $L / L^{I}$ is a totally ramified extension, one has that $\mathbb{F}_{L}=\mathbb{F}_{L^{I}}$ is still a finite field. The Inf-Res sequence reads as

$$
H^{1}\left(L^{I} / E, \bar{A}\left(\mathbb{F}_{L^{I}}\right)\right) \hookrightarrow H^{1}\left(L / E, \bar{A}\left(\mathbb{F}_{L}\right)\right) \rightarrow H^{1}\left(L / L^{I}, \bar{A}\left(\mathbb{F}_{L}\right)\right)
$$

The group on the left is obviously finite and for the one on the right we can use [13, Proposition 3.1] as done previously in Lemma 3.1 (because $I$ is still a $p$-adic Lie group) to get

$$
\left|H^{1}\left(L / L^{I}, \bar{A}\left(\mathbb{F}_{L}\right)\right)\right| \leqslant\left|\bar{A}\left(\mathbb{F}_{L}\right)\right|^{\tilde{d}_{1}}
$$

where the exponent $\tilde{d}_{1}=\tilde{d}_{1}(\operatorname{Gal}(L / E))$ only depends on $\operatorname{Gal}(L / E)$.

\subsection{Case 2: $I$ has infinite index in $\operatorname{Gal}(L / E)$.}

We use the same sequence

$$
H^{1}\left(L^{I} / E, \bar{A}\left(\mathbb{F}_{L^{I}}\right)\right) \hookrightarrow H^{1}\left(L / E, \bar{A}\left(\mathbb{F}_{L}\right)\right) \rightarrow H^{1}\left(L / L^{I}, \bar{A}\left(\mathbb{F}_{L}\right)\right)
$$

but now $\mathbb{F}_{L}$ is not a finite field anymore: indeed it contains the $\mathbb{Z}_{p}$-extension of $\mathbb{F}_{E}$ (because unramified extensions come from extensions of the field of constants). For the group on the right we again use Lemma 3.1 to prove that $H^{1}\left(L / L^{I}, \bar{A}\left(\mathbb{F}_{L}\right)\right)$ is a cofinitely generated $\mathbb{Z}_{p}$-module. The only difference with case $\mathbf{1}$ is that now the $p$ divisible part of $\bar{A}\left(\mathbb{F}_{L}\right)$ might come into play (moreover note that $H^{1}\left(L / L^{I}, \bar{A}\left(\mathbb{F}_{L}\right)\right)$ is a torsion abelian group, hence its $p$-primary part is exactly $H^{1}\left(L / L^{I}, \bar{A}\left(\mathbb{F}_{L}\right)\left[p^{\infty}\right]\right)$ and $A\left(\mathbb{F}_{L}\right)\left[p^{\infty}\right]$ has finite $\mathbb{Z}_{p}$-corank). For $H^{1}\left(L^{I} / E, \bar{A}\left(\mathbb{F}_{L^{I}}\right)\right)$ we observe that the $p$-part of $\operatorname{Gal}\left(L^{I} / E\right)$ is isomorphic to $\mathbb{Z}_{p}$ and that the subgroup of $\bar{A}\left(\mathbb{F}_{L^{I}}\right)$ fixed by that $p$-part is finite. Hence, by [5, Lemma 3.4 and Remark 3.5], one has that

$$
\left|H^{1}\left(L^{I} / E, \bar{A}\left(\mathbb{F}_{L^{I}}\right)\right)\right| \leqslant\left|\bar{A}\left(\mathbb{F}_{L^{I}}\right) /\left(\bar{A}\left(\mathbb{F}_{L^{I}}\right)\right)_{\operatorname{div}}\right|
$$

is finite.

The lemma provides a bound for the order of $H^{1}(L / E, A(L))$ which (when $I$ is open in $\operatorname{Gal}(L / E))$ can be written in terms of $\bar{A}\left[p^{\infty}\right]\left(\mathbb{F}_{E}\right)$ and $\bar{A}\left(\mathbb{F}_{L}\right)$. If the inertia is infinite (i.e., if $I$ has order divisible by arbitrary high powers of $p$ or, as we will say from now on, has order divisible by $p^{\infty}$ ) we can prove that the inflation map

$$
H^{1}\left(L / E, \widehat{A}\left(\mathcal{O}_{L}\right)\right) \hookrightarrow H^{1}\left(E, \widehat{A}\left(\mathcal{O}_{\bar{E}}\right)\right)
$$


is actually an isomorphism but, since this is not going to improve the bound, we decided to keep this statement out of the lemma and we include it here only for completeness (the proof is just a generalization of the one provided for [34, Theorem 2 (b)]).

Proposition 5.2. In the same setting of the previous lemma, assume that I has order divisible by $p^{\infty}$, then

$$
H^{1}\left(L / E, \widehat{A}\left(\mathcal{O}_{L}\right)\right)=H^{1}\left(E, \widehat{A}\left(\mathcal{O}_{\bar{E}}\right)\right)
$$

Proof. The inflation map immediately provides one inclusion so we need to prove the reverse one. By [34, Corollary 2.3.3], $H^{1}(L / E, A(L))$ is the annihilator of $N_{L / E}(B(L))$ with respect to the local Tate pairing (where $N_{L / E}$ is the natural norm map). This provides an isomorphism

$$
H^{1}(L / E, A(L)) \simeq\left(B(E) / N_{L / E}(B(L))\right)^{\vee} .
$$

Working as in [34, Section 2] (in particular Subsections 2.3 and 2.6 in which the results apply to general Galois extensions), one verifies that $H^{1}\left(E, \widehat{A}\left(\mathcal{O}_{\bar{E}}\right)\right)$ is the annihilator of $\widehat{B}\left(\mathcal{O}_{E}\right)$ with respect to the local pairing. Moreover, since $\widehat{A}\left(\mathcal{O}_{\bar{E}}\right)$ is the kernel of the reduction map, one has

$$
\begin{aligned}
H^{1}\left(E, \widehat{A}\left(\mathcal{O}_{\bar{E}}\right)\right) \cap H^{1}(L / E, A(L)) & \subseteq \operatorname{Ker}\left\{H^{1}(L / E, A(L)) \rightarrow H^{1}\left(L / E, \bar{A}\left(\mathbb{F}_{L}\right)\right)\right\} \\
& =H^{1}\left(L / E, \widehat{A}\left(\mathcal{O}_{L}\right)\right) .
\end{aligned}
$$

Therefore it suffices to show $H^{1}\left(E, \widehat{A}\left(\mathcal{O}_{\bar{E}}\right)\right) \subseteq H^{1}(L / E, A(L))$ and, because of the isomorphism (5.1), this is equivalent to proving $N_{L / E}(B(L)) \subseteq \widehat{B}\left(\mathcal{O}_{E}\right)$.

Take $\alpha \in B(L)$ and put $x=N_{L / E}(\alpha)$ : we can assume that $\alpha$ belongs to the $p$-part of $B(L)$, so that $x$ is in the $p$-part of $B(E)$. Let $E^{\prime}$ be an intermediate field containing $L^{I}$ and such that $p^{\infty}$ divides $\left[E^{\prime}: L^{I}\right]$. Consider $z=N_{L / E^{\prime}}(\alpha) \in B\left(E^{\prime}\right)$ and let $\bar{z}$ be the image of $z$ in $\bar{B}\left(\mathbb{F}_{E^{\prime}}\right)=\bar{B}\left(\mathbb{F}_{L^{I}}\right)$ (which is a torsion group). Hence $\bar{z}$ has finite order, say $p^{m}$, and we can find a field $E^{\prime \prime}$ between $E^{\prime}$ and $L^{I}$ such that $p^{m} \mid\left[E^{\prime \prime}: L^{I}\right]$. Put $y=N_{L / E^{\prime \prime}}(\alpha)=N_{E^{\prime} / E^{\prime \prime}}(z) \in B\left(E^{\prime \prime}\right)$, so that $x=N_{E^{\prime \prime} / E}(y)$ and note that $\bar{y}$ (the image of $y$ in $\bar{B}\left(\mathbb{F}_{E^{\prime \prime}}\right)=\bar{B}\left(\mathbb{F}_{L^{I}}\right)$ ) has order dividing $p^{m}$. Since $\operatorname{Gal}\left(E^{\prime \prime} / L^{I}\right)$ fixes $\bar{y}$, one has that $N_{E^{\prime \prime} / L^{I}}(\bar{y})$ is trivial in $\bar{B}\left(\mathbb{F}_{L^{I}}\right)$, i.e., $N_{E^{\prime \prime} / L^{I}}(y) \in$ $\widehat{B}\left(\mathcal{O}_{L^{I}}\right)$ (which is the kernel of the reduction map). Hence

$$
x=N_{E^{\prime \prime} / E}(y)=N_{L^{I} / E}\left(N_{E^{\prime \prime} / L^{I}}(y)\right) \in N_{L^{I} / E}\left(\widehat{B}\left(\mathcal{O}_{L^{I}}\right)\right) \subseteq \widehat{B}\left(\mathcal{O}_{E}\right) .
$$

Now we proceed with our control theorem.

Theorem 5.3. Assume that all ramified primes are of good ordinary or split multiplicative reduction. Then, for any finite extension $F^{\prime} / F$ contained in $K$, the kernels and cokernels of the maps

$$
a_{K / F^{\prime}}: \operatorname{Sel}_{A}\left(F^{\prime}\right)_{p} \rightarrow \operatorname{Sel}_{A}(K)_{p}^{\mathrm{Gal}\left(K / F^{\prime}\right)}
$$


are cofinitely generated $\mathbb{Z}_{p}$-modules. If all primes in $S$ and all primes of bad reduction have decomposition groups open in $G$, then the coranks of kernels and cokernels are bounded independently of $F^{\prime}$. Moreover if the group $A\left[p^{\infty}\right](K)$ is finite, all places in $S$ are of good reduction and have inertia groups open in their decomposition groups, then the kernels and cokernels are finite (of bounded order if the primes of bad reduction have open decomposition group).

Proof. We work with the usual diagram (2.1), where now we cannot substitute flat cohomology with Galois cohomology and in the groups $\mathcal{G}_{A}(\cdot)$ the images of the local Kummer maps are nontrivial (in general).

Since the map $\mathcal{X}_{K} \rightarrow \mathcal{X}_{F^{\prime}}$ is a Galois covering with Galois group $\operatorname{Gal}\left(K / F^{\prime}\right)$, the Hochschild-Serre spectral sequence applies and we will study:

- $\operatorname{Ker}\left(b_{K / F^{\prime}}\right) \simeq H^{1}\left(K / F^{\prime}, A\left[p^{\infty}\right](K)\right)$;

- $\operatorname{Coker}\left(b_{K / F^{\prime}}\right) \subseteq H^{2}\left(K / F^{\prime}, A\left[p^{\infty}\right](K)\right)$;

- $\operatorname{Ker}\left(c_{K / F^{\prime}}\right)$

noting that the cohomology groups on the right are Galois cohomology groups (see [20, III.2.21 (a), (b) and III.1.17 (d)]).

\subsection{The map $b_{K / F^{\prime}}$}

Just use Lemma 3.1 .

\subsection{The maps $c_{K / F^{\prime}}$}

As before we simply work with the maps

$$
d_{w}: H_{f l}^{1}\left(X_{F_{v^{\prime}}^{\prime}}, A\left[p^{\infty}\right]\right) / \operatorname{Im} \kappa_{v^{\prime}} \rightarrow H_{f l}^{1}\left(X_{K_{w}}, A\left[p^{\infty}\right]\right) / \operatorname{Im} \kappa_{w} .
$$

From the Kummer sequence one gets a diagram

$$
\begin{aligned}
& H_{f l}^{1}\left(X_{F_{v^{\prime}}^{\prime}}, A\left[p^{\infty}\right]\right) / \operatorname{Im} \kappa_{v^{\prime}} \longrightarrow H_{f l}^{1}\left(X_{F_{v^{\prime}}^{\prime}}, A\right)\left[p^{\infty}\right] \\
& H_{f l}^{1}\left(X_{K_{w}}, A\left[p^{\infty}\right]\right) / \operatorname{Im} \kappa_{w} \smile \longrightarrow H_{f l}^{1}\left(X_{K_{w}}, A\right)\left[p^{\infty}\right] \text {, }
\end{aligned}
$$

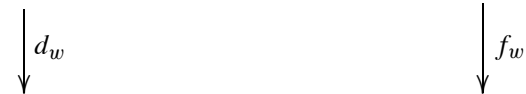

and (from the Inf-Res sequence)

$$
\operatorname{Ker}\left(d_{w}\right) \hookrightarrow \operatorname{Ker}\left(f_{w}\right) \simeq H^{1}\left(K_{w} / F_{v^{\prime}}^{\prime}, A\left(K_{w}\right)\right)\left[p^{\infty}\right]
$$

Before moving on observe that $K_{w} / F_{v^{\prime}}^{\prime}$ is a $p$-adic Lie extension because the decomposition group of any place is closed in $\operatorname{Gal}\left(K / F^{\prime}\right)$. Now we distinguish two cases depending on their behaviour in $K / F^{\prime}$ (and as usual, we do not consider primes which split completely because they give no contribution to $\operatorname{Ker}\left(c_{K / F^{\prime}}\right)$ ). 


\subsubsection{Unramified primes}

If $v^{\prime}$ is unramified, from [21, Proposition I.3.8], we have

$$
H^{1}\left(\left(F_{v^{\prime}}^{\prime}\right)^{\mathrm{unr}} / F_{v^{\prime}}^{\prime}, A\left(\left(F_{v^{\prime}}^{\prime}\right)^{\mathrm{unr}}\right)\right)=H^{1}\left(\left(F_{v^{\prime}}^{\prime}\right)^{\mathrm{unr}} / F_{v^{\prime}}^{\prime}, \pi_{0}\left(\mathcal{A}\left(F_{v^{\prime}}^{\prime}\right)_{0}\right)\right)
$$

where $\pi_{0}\left(\mathcal{A}\left(F_{v^{\prime}}^{\prime}\right)_{0}\right)$ is the set of connected components of the closed fiber of the Néron model $\mathcal{A}\left(F_{v^{\prime}}^{\prime}\right)$ of $A$ at $v^{\prime}$. The latter is a finite module and it is trivial when $v^{\prime}$ is a place of good reduction. Because of the inflation map

$$
H^{1}\left(K_{w} / F_{v^{\prime}}^{\prime}, A\left(K_{w}\right)\right) \hookrightarrow H^{1}\left(\left(F_{v^{\prime}}^{\prime}\right)^{\mathrm{unr}} / F_{v^{\prime}}^{\prime}, A\left(\left(F_{v^{\prime}}^{\prime}\right)^{\mathrm{unr}}\right)\right)
$$

the same holds for $\operatorname{Ker}\left(d_{w}\right)$ as well. Note also that in finite unramified (hence cyclic) extensions, since the group $\pi_{0}\left(\mathcal{A}\left(F_{v^{\prime}}^{\prime}\right)_{0}\right)$ is finite, the order of the $H^{1}$ is equal to the order of the $H^{0}$ which is uniformly bounded (see for example [34, Lemma 3.3.1]). Thus the order of $H^{1}\left(\left(F_{v^{\prime}}^{\prime}\right)^{\mathrm{unr}} / F_{v^{\prime}}^{\prime}, \pi_{0}\left(\mathcal{A}\left(F_{v^{\prime}}^{\prime}\right)_{0}\right)\right)$ is bounded independently of $F^{\prime}$.

\subsubsection{Ramified primes}

If $v^{\prime}$ is of good ordinary reduction, just observe that $\operatorname{Gal}\left(K_{w} / F_{v^{\prime}}^{\prime}\right)$ is a $p$-adic Lie group with nontrivial inertia and apply Lemma 5.1.

We are left with ramified primes of split multiplicative reduction. Let $v^{\prime}$ be such a prime. We have the exact sequence coming from Mumford's uniformization

$$
<q_{A, 1}^{\prime}, \ldots, q_{A, g}^{\prime}>\hookrightarrow\left(\left(\overline{F_{v}}\right)^{*}\right)^{g} \rightarrow A\left(\overline{F_{v}}\right)
$$

(where we recall that $g$ is the dimension of the variety $A$, the $q_{A, i}^{\prime}$ are parameters in $\left(F_{v^{\prime}}^{\prime}\right)^{*}$ and the morphisms behave well with respect to the Galois action).

Taking cohomology (and using Hilbert's Theorem 90) one finds an injection

$$
H^{1}\left(K_{w} / F_{v^{\prime}}^{\prime}, A\left(K_{w}\right)\right) \hookrightarrow H^{2}\left(K_{w} / F_{v^{\prime}}^{\prime},<q_{A, 1}^{\prime}, \ldots, q_{A, g}^{\prime}>\right) .
$$

Since $q_{A, i}^{\prime} \in\left(F_{v^{\prime}}^{\prime}\right)^{*}$, the action of the Galois group is trivial on them and we have an isomorphism of Galois modules $<q_{A, 1}^{\prime}, \ldots, q_{A, g}^{\prime}>\simeq \mathbb{Z}^{g}$. Hence

$$
H^{2}\left(K_{w} / F_{v^{\prime}}^{\prime},<q_{A, 1}^{\prime}, \ldots, q_{A, g}^{\prime}>\right) \simeq H^{2}\left(K_{w} / F_{v^{\prime}}^{\prime}, \mathbb{Z}^{g}\right) \simeq\left(\left(\operatorname{Gal}\left(K_{w} / F_{v^{\prime}}^{\prime}\right)^{a b}\right)^{\vee}\right)^{g}
$$

(where the last one is the Pontrjagin dual of the maximal abelian quotient of $\left.\operatorname{Gal}\left(K_{w} / F_{v^{\prime}}^{\prime}\right)\right)$. The last one is a cofinitely generated $\mathbb{Z}_{p}$-module, since $\mathrm{Gal}\left(K_{w} / F_{v^{\prime}}^{\prime}\right)^{a b}$ is virtually a finitely generated $\mathbb{Z}_{p}$-module. Indeed, $\overline{\left[\operatorname{Gal}\left(K_{w} / F_{v^{\prime}}^{\prime}\right), \operatorname{Gal}\left(K_{w} / F_{v^{\prime}}^{\prime}\right)\right]}$ (the topological closure of the commutators) is a closed normal subgroup of $\operatorname{Gal}\left(K_{w} / F_{v^{\prime}}^{\prime}\right)$ and their quotient is still a $p$-adic Lie group (see [11, Theorem 9.6 (ii)] and, for the $\mathbb{Z}_{p}$-module structure, [11, Theorem 4.9] and [11, Theorem 4.17]). 


\section{Remark 5.4.}

1. The hypothesis on the reduction of $\mathrm{A}$ at ramified primes is necessary. Indeed, if $v$ is a ramified prime of good supersingular reduction and $\operatorname{Gal}(K / F) \simeq \mathbb{Z}_{p}^{d}$ (a deeply ramified extension in the sense of [10, Section 2]), then K.-S. Tan has shown that $H^{1}\left(K_{w} / F_{v^{\prime}}^{\prime}, A\left(K_{w}\right)\right)\left[p^{\infty}\right]$ has infinite $\mathbb{Z}_{p}$-corank (see [35, Theorem 3.10]).

2. When $A=E$ is an elliptic curve it is easy to see that the number of torsion points of $p$-power order in a separable extension of $F$ is finite (see, for example, [4, Lemma 4.3]). For a general abelian variety K.-S. Tan has proved similar results for local fields in [34, Lemma 2.5.1] and for $\mathbb{Z}_{p}^{d}$-extensions of global fields (in [35, Proposition 2.11]). Hence, for a $p$-adic Lie extension, we are interested only in the number of $p$-power torsion points in $\mathbb{F}_{p}^{(p)} F$ (or $\mathbb{F}_{p}^{(p)} F^{\prime}$ for some finite unramified extension $F^{\prime}$ of $F$ ).

\section{6. $\Lambda$-modules for $\ell=p$}

The Selmer groups are again $\Lambda(G)$-modules and we will investigate their structure as in the case $l \neq p$. We assume that $G$ does not contain any element of order $p$.

\subsection{Structure of $\operatorname{Sel}_{A}(K)_{p}^{\vee}$ as $\Lambda(G)$-module}

Theorem 6.1. With the above notations, if all places in $S$ are of good ordinary or split multiplicative reduction, then $\operatorname{Sel}_{A}(K)_{p}^{\vee}$ is a finitely generated $\Lambda(G)$-module.

Proof. This is the same proof of Theorem 4.3, using Theorems 5.3 and 4.1.

Theorem 6.2. Assume that there exists an open soluble, uniform and pro-p subgroup $G^{\prime}$ of $G$. Suppose that $A\left[p^{\infty}\right](K)$ and $\operatorname{Sel}_{A}\left(F^{\prime}\right)_{p}^{\vee}$, where $F^{\prime}$ is the fixed field of $G^{\prime}$, are finite. If all ramified places of $F$ are of good ordinary reduction for $A$ and have inertia groups open in their decomposition groups, then $\operatorname{Sel}_{A}(K)_{p}^{\vee}$ is a torsion $\Lambda(G)$-module.

Proof. It is sufficient to show that $\operatorname{Sel}_{A}(K)_{p}^{\vee}$ is a torsion $\Lambda\left(G^{\prime}\right)$-module (classical meaning). In order to do this just use Theorems 5.3 and 4.1.

\subsection{Structure of $\operatorname{Sel}_{A}(K)_{p}^{\vee}$ as $\Lambda(H)$-module}

Assume that there exists a closed normal subgroup $H$ in $G$ such that $G / H=\Gamma \simeq$ $\mathbb{Z}_{p}$ and let $K^{\prime}$ be its fixed field.

We need the following:

Lemma 6.3. With notations as above, let $K^{\prime}:=K^{H}$ be the fixed field of $H$. If $v \in \Sigma_{F}$ is unramified in $K / F$, then

1. $v$ splits completely in $K^{\prime} / F$ or

2. the decomposition group, in $H=\mathrm{Gal}\left(K / K^{\prime}\right)$, of any prime $w^{\prime}$ of $K^{\prime}$ dividing $v$ is finite and has order prime to $p$. 
Proof. Consider the diagram

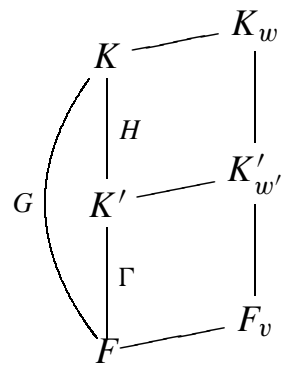

where $w\left|w^{\prime}\right| v$.

Assume $v$ is unramified, then, $\operatorname{since} \operatorname{Gal}\left(K_{w} / F_{v}\right)$ is still a $p$-adic Lie group and the $p$-part of an unramified $p$-adic Lie extension of local fields is at most a $\mathbb{Z}_{p}$-extension, we have to deal with two cases.

a. $\operatorname{Gal}\left(K_{w} / F_{v}\right)$ is finite. Then $\operatorname{Gal}\left(K_{w^{\prime}}^{\prime} / F_{v}\right)$ is a finite subgroup of $\Gamma \simeq \mathbb{Z}_{p}$, hence it is trivial and $v$ splits completely if $K^{\prime} / F$ (i.e., 1 holds).

b. $\operatorname{Gal}\left(K_{w} / F_{v}\right) \simeq \Gamma$. Then $\operatorname{Gal}\left(K_{w^{\prime}}^{\prime} / F_{v}\right)$ can be trivial or isomorphic to $\Gamma$. If it is trivial we are back to case 1 . If it is $\Gamma$, then $K_{w^{\prime}}^{\prime}$ is the unramified $\mathbb{Z}_{p}$-extension of $F_{v}$. Hence the extension $K_{w} / K_{w^{\prime}}^{\prime}$ is unramified of (finite) order prime to $p$ (i.e., 2 holds).

As in the $\ell \neq p$ case, we will show a slightly modified version of Theorem 5.3.

Theorem 6.4. Assume that:

1. all places in $S$ are of split multiplicative reduction for $A$;

2. all places in $S$ and all places of bad reduction for A split in finitely many primes in $K^{\prime} / F$.

Then the map

$$
a: \operatorname{Sel}_{A}\left(K^{\prime}\right)_{p} \rightarrow \operatorname{Sel}_{A}(K)_{p}^{H}
$$

has cofinitely generated kernel and cokernel (viewed as $\mathbb{Z}_{p}$-modules).

Proof. We go directly to the local kernels for places which do not split completely

$$
\operatorname{Ker}\left(d_{w}\right) \simeq H^{1}\left(K_{w} / K_{w^{\prime}}^{\prime}, A\left(K_{w}\right)\right)\left[p^{\infty}\right] .
$$

\subsubsection{Unramified primes of good reduction}

Let $v$ be an unramified prime of good reduction for $A$. From Lemma 6.3 we know that $K_{w^{\prime}}^{\prime}=F_{v}$ is a local field or $\operatorname{Gal}\left(K_{w} / K_{w^{\prime}}^{\prime}\right)$ has finite order prime with $p$. In the first case [21, Chapter I, Proposition 3.8] shows that $H^{1}\left(K_{w} / K_{w^{\prime}}^{\prime}, A\left(K_{w}\right)\right)=$ 0 and we get our claim. In the second case the $p$-part of the torsion module $H^{1}\left(K_{w} / K_{w^{\prime}}^{\prime}, A\left(K_{w}\right)\right)$ is obviously trivial.

Because of our hypothesis on the splitting of primes we are already left with finitely many local kernels, now we check the behaviour of the remaining ones. 


\subsubsection{Unramified primes of bad reduction}

As above if $\operatorname{Gal}\left(K_{w^{\prime}}^{\prime} / F_{v}\right) \simeq \Gamma$ (i.e., $K_{w^{\prime}}^{\prime}$ is not a local field), then the $p$-part of $H^{1}\left(K_{w} / K_{w^{\prime}}^{\prime}, A\left(K_{w}\right)\right)$ is trivial. The other case (i.e., $\left.K_{w^{\prime}}^{\prime}=F_{v}\right)$ cannot happen because we are assuming that bad reduction primes do not split completely in $K^{\prime}$.

\subsubsection{Ramified primes}

For the ramified ones we use Mumford's parametrization as in Section 5.4.2. First we get an exact sequence

$$
<q_{A, 1}, \ldots, q_{A, g}>\hookrightarrow\left(K_{w}^{*}\right)^{g} \rightarrow A\left(K_{w}\right)
$$

(where we can always assume that the periods are in the base field $F_{v}$ ). Then $\operatorname{Gal}\left(K_{w} / K_{w^{\prime}}^{\prime}\right)$-cohomology provides an injection

$$
H^{1}\left(K_{w} / K_{w^{\prime}}^{\prime}, A\left(K_{w}\right)\right) \hookrightarrow H^{2}\left(K_{w} / K_{w^{\prime}}^{\prime}, \mathbb{Z}^{g}\right) \simeq\left(\left(\operatorname{Gal}\left(K_{w} / K_{w^{\prime}}^{\prime}\right)^{a b}\right)^{\vee}\right)^{g}
$$

As a consequence we have the following (which generalizes [23, Theorem 1.9]).

Corollary 6.5. In the setting of Theorem 6.4, assume that $\operatorname{Sel}_{A}\left(K^{\prime}\right)_{p}^{\vee}$ is a finitely generated $\mathbb{Z}_{p}$-module. Then $\operatorname{Sel}_{A}(K)_{p}^{\vee}$ is finitely generated over $\Lambda(H)$ (hence torsion over $\Lambda(G)$ ).

\subsection{Final summary}

Take the function fields $K$ and $K^{\prime}$ such that the hypothesis on the splitting of primes in Theorem 5.3 is verified.

For $\operatorname{Sel}_{A}(K)_{p}^{\vee}$ to be finitely generated as $\Lambda(G)$-module we need just to assume that all primes in $S$ are of good ordinary or split multiplicative reduction for $A$. To move a step further and find $\Lambda(G)$-torsion modules we can assume

1. all ramified primes are of good ordinary reduction and have open inertia groups;

2. $A\left[p^{\infty}\right](K)$ and $\operatorname{Sel}_{A}\left(F^{\prime}\right)_{p}^{\vee}$ are finite (where $F^{\prime}$ is the fixed field of an open soluble, uniform and pro- $p$ subgroup $G^{\prime}$ of $G$, if such a subgroup exists),

and use Theorem 6.2.

Another way to find $\Lambda(G)$-torsion modules is provided by Corollary 6.5. It somehow complements the previous one because it requires a different type of reduction for the ramified places. The assumptions for this case are:

3. all ramified primes are of split multiplicative reduction;

4. $\operatorname{Sel}_{A}\left(K^{\prime}\right)_{p}$ is cofinitely generated over $\mathbb{Z}_{p}$.

One example for this second case is given by the usual arithmetic extension $K^{\prime}=$ $\mathbb{F}_{p}^{(p)} F$ for which the hypothesis $\mathbf{1}$ above obviously does not hold and the hypothesis $\mathbf{4}$ is proved (in some cases) in [23, Theorem 1.8]. 
Remark 6.6. We found conditions to get $\Lambda(G)$-torsion modules for different types of reduction for the ramified primes, but we remind that, to get a characteristic element for a $\Lambda(G)$-torsion module for a non-commutative group $G$, one needs to examine the $\Lambda(H)$-module structure as well. Hence our results provide characteristic elements for $\operatorname{Sel}_{A}(K)_{p}^{\vee}$ only when the ramified primes are of split multiplicative reduction.

\section{References}

[1] S. ARIAS-DE-ReYna, W. GAJDA and S. PETERSEn, Big monodromy theorem for abelian varieties over finitely generated fields, J. Pure Appl. Algebra 217 (2013), 218-229.

[2] P. N. BALISTER and S. HowsON, Note on Nakayama's lemma for compact $\Lambda$-modules, Asian J. Math. 1 (1997), 224-229.

[3] A. BAndini, F. BARS and I. LONGHI, Aspects of Iwasawa theory over function fields, arXiv:1005.2289v2 [math.NT], EMS Congress Reports, to appear.

[4] A. BANDINI and I. LONGHI, Control Theorems for elliptic curves over function fields, Int. J. Number Theory 5 (2009), 229-256.

[5] A. BANDINI and I. LONGHI, Selmer groups for elliptic curves in $\mathbb{Z}_{l}^{d}$-extensions of function fields of characteristic p, Ann. Inst. Fourier (Grenoble) 59 (2009), 2301-2327.

[6] A. BANDINI, I. LONGHI and S. VIGNI, Torsion points on elliptic curves over function fields and $a$ theorem of Igusa, Expo. Math. 27 (2009), 175-209.

[7] A. BANDINI and M. VALENTINO, On Selmer groups of abelian varieties over $\ell$-adic Lie extensions of global function fields, Bull. Braz. Math. Soc. (N.S.) 45 (2014), 575-595.

[8] D. BURNS and O. VENJAKOB, On descent theory and main conjectures in non-commutative Iwasawa theory, J. Inst. Math. Jussieu 10 (2011), 59-118.

[9] J. Coates, T. Fukaya, K. Kato, R. Sujatha and O. Venjakob, The GL2 main conjecture for elliptic curves without complex multiplication, Publ. Math. Inst. Hautes Études Sci. 101 (2005), 163-208.

[10] J. CoATES and R. GREENBERG, Kummer theory for abelian varieties over local fields, Invent. Math. 124 (1996), 129-174.

[11] J. D. Dixon, M. P. F. DU Sautoy, A. Mann and D. Segal, "Analytic Pro- $p$ Groups" 2nd Edition, Cambridge Studies in Advanced Mathematics, Vol. 61, Cambridge Univ. Press, 1999.

[12] J. S. EllenBERG, Selmer groups and Mordell-Weil groups of elliptic curves over towers of function fields, Compos. Math. 142 (2006), 1215-1230.

[13] R. GREEnBERG, Galois Theory for the Selmer Group of an Abelian Variety, Compos. Math. 136 (2003), 255-297.

[14] C. HALL, An open image theorem for a general class of abelian varieties. With an appendix by Emmanuel Kowalski, Bull. Lond. Math. Soc. 43 (2011), 703-711.

[15] K. KATO and F. TRIHAN, On the conjectures of Birch and Swinnerton-Dyer in characteristic $p>0$, Invent. Math. 153 (2003), 537-592.

[16] K. F. LAI, I. LONGHI, K.-S. TAN and F. TRIHAN, The Iwasawa main conjecture of constant ordinary abelian varieties over function fields, arXiv:1406.6125 [math. NT].

[17] M. LAZARD, Groupes analytiques p-adiques, Publ. Math. Inst. Hautes Études Sci. 26 (1965), 389-603.

[18] A. Lubotzky, A. Mann and D. Segal, Finitely generated groups of polynomial subgroup growth, Israel J. Math. 82 (1993), 363-371.

[19] B. MAZUR, Rational points of abelian varieties with values in towers of number fields, Invent. Math. 18 (1972), 183-266. 
[20] J. S. Milne, "Étale Cohomology”, Princeton Mathematical Series, Vol. 33, Princeton University Press, Princeton, NJ, 1980.

[21] J. S. Milne, "Arithmetic Duality Theorems", BookSurge, LLC, Second edition, 2006.

[22] J. NeuCHIRCH, A. SCHMidT and K. WingBerG, "Cohomology of number fields" Second edition, GTM 323, Springer-Verlag, 2008.

[23] T. OCHIAI and F. TRIHAN, On the Selmer groups of abelian varieties over function fields of characteristic $p>0$, Math. Proc. Cambridge Philos. Soc. 146 (2009), 23-43.

[24] A. PACHECO, Selmer groups of abelian varieties in extensions of function fields, Math. Z. 261 (2009), 787-804

[25] A. PAL, Functional equation of characteristic elements of abelian varieties over function fields $\ell \neq p$, Int. J. Number Theory 10 (2014), 705-735.

[26] P. SCHNEIDER, Zur Vermutung von Birch und Swinnerton-Dyerber globalen Funktionenkrpern (German) [On the conjecture of Birch and Swinnerton-Dyer over global function fields], Math. Ann. 260 (1982), 495-510.

[27] G. SECHI, " $G L_{2}$ Iwasawa Theory of Elliptic Curves over Global Function Fields", PhD thesis, University of Cambridge, 2006.

[28] J.-P. SERRE, Sur la dimension cohomologique des groupes profinis, Topology 3 (1965), 413-420.

[29] J.-P. SERRE, Sur les groupes de congruence des variétés abéliennes II, Izv. Akad. Nauk SSSR, Ser. Mat. 35 (1971), 731-737.

[30] J.-P. SERRE, "Cohomologie Galoisienne", Lecture Notes in Mathematics, Vol. 5, SpringerVerlag, 1994.

[31] J.-P. SERRE and J. TATE, Good reduction of abelian varieties, Ann. of Math. 88 (1968), 492-517.

[32] J. H. Silverman, "The Arithmetic of Elliptic Curves", 2nd Edition, GTM 106, Springer, 1986.

[33] J. H. Silverman, "Advanced Topics in the Arithmetic of Elliptic Curves", GTM 151, Springer-Verlag, New York, 1994.

[34] K. S. TAN, A generalized Mazur's theorem and its applications, Trans. Amer. Math. Soc. 362 (2010), 4433-4450.

[35] K. S. TAN, Selmer groups over $\mathbb{Z}_{p}^{d}$-extensions, Math. Ann. 359 (2014), 1025-1075.

[36] O. VenJaKob, On the structure theory of the Iwasawa algebra of a p-adic Lie group, $\mathrm{J}$. Eur. Math. Soc. (JEMS) 4 (2002), 271-311.

[37] J. S. WILSON, "Profinite Groups", London Mathematical Society Monographs, New Series, 19, The Clarendon Press, Oxford University Press, New York, 1998.

[38] M. WiTtE, On a noncommutative Iwasawa main conjecture for varieties over finite fields, J. Eur. Math. Soc. (JEMS) 16 (2014), 289-325.

Università degli Studi di Parma

Dipartimento di Matematica

Parco Area delle Scienze, 53/A

43124 Parma - Italia

andrea.bandini@unipr.it

Università della Calabria

Dipartimento di Matematica e Informatica

Via P. Bucci - Cubo 31B

87036 Arcavacata di Rende (CS) - Italia

valentino@mat.unical.it 